\title{
ENTROPY AND APPROXIMATION NUMBERS OF EMBEDDINGS OF FUNCTION SPACES WITH MUCKENHOUPT WEIGHTS, II. GENERAL WEIGHTS
}

\author{
Dorothee D. Haroske* and Leszek Skrzypczak
}

\author{
Friedrich-Schiller-University Jena, Mathematical Institute \\ D-07737 Jena, Germany; dorothee.haroske@uni-jena.de \\ Adam Mickiewicz University, Faculty of Mathematics and Computer Science \\ Ul. Umultowska 87, 61-614 Poznań, Poland; lskrzyp@amu.edu.pl
}

\begin{abstract}
We study compact embeddings for weighted spaces of Besov and Triebel-Lizorkin type where the weights belong to Muckenhoupt $\mathscr{A}_{p}$ classes. We focus our attention on the influence of singular points of the weights on the compactness of the embeddings as well as on the asymptotic behaviour of their entropy and approximation numbers.
\end{abstract}

\section{Introduction}

In recent years some attention has been paid to the compactness of embeddings of weighted function spaces of Besov and Sobolev (or, more generally, Triebel-Lizorkin) type as well as to analytic and geometric quantities describing this compactness, in particular, corresponding approximation and entropy numbers. The investigations in the above described context started by the work of the first named author and Triebel, cf. $[15,19]$ (note that there is a partial forerunner in [27, Ch. V, §3, Thm. 9]), and were continued and extended by Kühn, Leopold, Sickel and the second author in the series of papers $[21,22,23,33]$. As an application one obtains spectral estimates of certain pseudo-differential operators in the way of the program proposed by Edmunds and Triebel [10].

In all above papers the class of so-called 'admissible' weights was considered. These are smooth weights with no singular points. One can take $w(x)=\left(1+|x|^{2}\right)^{\alpha / 2}$, $\alpha \in \mathbf{R}, x \in \mathbf{R}^{n}$, as a prominent example. Developing these investigations the authors started in [17] to consider similar problems for weights from the Muckenhoupt class $\mathscr{A}_{\infty}$. In contrast to 'admissible' weights the $\mathscr{A}_{\infty}$ weights may have local singularities which can influence properties of the embeddings of function spaces. Now, the weight $w(x)=|x|^{\alpha}, \alpha>-n$, may serve as a typical example. In [17] we obtained necessary and sufficient conditions on the parameters and weights of the Besov spaces which guarantee continuity and compactness of such embeddings. Moreover, the exact asymptotic behaviour of entropy and approximation numbers of the embeddings of the spaces with weights that have purely polynomial growth, both near some singular point and at infinity, is determined. Our method in [17] essentially relies on a wavelet

doi:10.5186/aasfm.2011.3607

2010 Mathematics Subject Classification: Primary 46E35, 42B35, 41A46, 47B06.

Key words: Function spaces, Muckenhoupt weights, compact embeddings, entropy numbers, approximation numbers, singularities.

*The research was partially supported by the program 'DAAD-MEN' D/06/25518 and also by the DFG Heisenberg fellowship HA 2794/1. 
decomposition in weighted spaces which admits to deal with weighted sequence spaces instead of weighted function spaces in the sequel.

In this paper we study general $\mathscr{A}_{\infty}$ weights with a bounded set of singularities. We observed in [17] that in case of Muckenhoupt weights of polynomial type such singularities have influence on the compactness of the embeddings, but not on the asymptotic behaviour of their entropy and approximation numbers. The main aim of this paper is to prove a similar phenomenon for general $\mathscr{A}_{\infty}$ weights with a bounded set of singularities $\mathbf{S}_{\text {sing }}(w)$ : it turns out that concerning embeddings of spaces on $\mathbf{R}^{n}$, only the behaviour of the weight at infinity together with properties of the embeddings of function spaces on bounded sets are decisive for the asymptotic behaviour of their entropy and approximation numbers. In particular, we can prove the following result. Let $w \in \mathscr{A}_{\infty}$ with $r_{w}:=\inf \left\{r \geq 1: w \in \mathscr{A}_{r}\right\}$, and the set $\mathbf{S}_{\text {sing }}(w)$ of singular points of the weight $w$ be bounded. If

$$
s_{1}-\frac{n}{p_{1}}-s_{2}+\frac{n}{p_{2}}>n \max \left(\frac{1}{p_{2}}-\frac{1}{p_{1}}, 0\right)+\frac{n}{p_{1}}\left(r_{w}-1\right),
$$

and $Q$ is some sufficiently large cube with $\overline{\mathbf{S}_{\text {sing }}(w)} \subsetneq \stackrel{Q}{Q}$, then both

$$
\mathrm{id}_{w}^{Q}: A_{p_{1}, q_{1}}^{s_{1}}(Q, w) \hookrightarrow A_{p_{2}, q_{2}}^{s_{2}}(Q) \quad \text { and } \quad \operatorname{id}_{w w}^{Q}: A_{p_{1}, q_{1}}^{s_{1}}(Q, w) \hookrightarrow A_{p_{2}, q_{2}}^{s_{2}}(Q, w)
$$

are compact with

$$
e_{k}\left(\operatorname{id}_{w}^{Q}\right) \sim e_{k}\left(\operatorname{id}_{w w}^{Q}\right) \sim k^{-\frac{s_{1}-s_{2}}{n}}, \quad k \in \mathbf{N}
$$

where $A_{p_{i}, q_{i}}^{s_{i}}(Q, w)$ and $A_{p_{i}, q_{i}}^{s_{i}}(Q)$ stand for (un)weighted spaces of Besov or TriebelLizorkin type on $Q$. Consequently, the behaviour of entropy numbers is the same as in the unweighted case. There is a parallel result for the corresponding approximation numbers.

The paper is organized as follows. In Section 2 we recall basic facts about Muckenhoupt weights and weighted spaces needed later on. Section 3 is devoted to the continuity and compactness of the embeddings. In the last section we introduce the set $\mathbf{S}_{\text {sing }}\left(w_{1}, w_{2}\right)$ of singular points of the weights $w_{1}, w_{2} \in \mathscr{A}_{\infty}$. We prove that this set is of measure zero and finally determine the exact asymptotic behaviour of the entropy and approximation numbers for weights with a bounded set of singularities. We illustrate this concept with some well-known examples.

\section{Weighted function spaces}

First of all we need to fix some notation. By $\mathbf{N}$ we denote the set of natural numbers, by $\mathbf{N}_{0}$ the set $\mathbf{N} \cup\{0\}$, and by $\mathbf{Z}^{n}$ the set of all lattice points in $\mathbf{R}^{n}$ having integer components. The positive part of a real function $f$ is given by $f_{+}(x)=$ $\max (f(x), 0)$. If $0<u \leq \infty$, the number $u^{\prime}$ is given by $\frac{1}{u^{\prime}}=\left(1-\frac{1}{u}\right)_{+}$. For two positive real sequences $\left\{\alpha_{k}\right\}_{k \in \mathbf{N}}$ and $\left\{\beta_{k}\right\}_{k \in \mathbf{N}}$ we mean by $\alpha_{k} \sim \beta_{k}$ that there exist constants $c_{1}, c_{2}>0$ such that $c_{1} \alpha_{k} \leq \beta_{k} \leq c_{2} \alpha_{k}$ for all $k \in \mathbf{N}$; similarly, for positive functions.

Given two (quasi-)Banach spaces $X$ and $Y$, we write $X \hookrightarrow Y$ if $X \subset Y$ and the natural embedding of $X$ in $Y$ is continuous.

All unimportant positive constants will be denoted by $c$, occasionally with subscripts. For convenience, let both $d x$ and $|\cdot|$ stand for the ( $n$-dimensional) Lebesgue measure in the sequel. If not otherwise indicated, log is always taken with respect to base 2 . 
2.1. Muckenhoupt weights. We briefly recall some fundamentals on Muckenhoupt classes $\mathscr{A}_{p}$.

Definition 2.1. Let $w$ be a positive, locally integrable function on $\mathbf{R}^{n}$, and $1<p<\infty$. Then $w$ belongs to the Muckenhoupt class $\mathscr{A}_{p}$, if there exists a constant $0<A<\infty$ such that for all balls $B$ the following inequality holds

$$
\left(\frac{1}{|B|} \int_{B} w(x) d x\right)^{1 / p} \cdot\left(\frac{1}{|B|} \int_{B} w(x)^{-p^{\prime} / p} d x\right)^{1 / p^{\prime}} \leq A,
$$

where $p^{\prime}$ is the dual exponent to $p$ given by $1 / p^{\prime}+1 / p=1$ and $|B|$ stands for the Lebesgue measure of the ball $B$.

The limiting cases $p=1$ and $p=\infty$ can be incorporated as follows. By a weight $w$ we shall always mean a locally integrable function $w \in L_{1}^{\operatorname{loc}}\left(\mathbf{R}^{n}\right)$, positive a.e. in the sequel. Let $M$ stand for the Hardy-Littlewood maximal operator given by

$$
M f(x)=\sup _{B(x, r) \in \mathscr{B}} \frac{1}{|B(x, r)|} \int_{B(x, r)}|f(y)| d y, \quad x \in \mathbf{R}^{n}
$$

where $\mathscr{B}$ is the collection of all open balls

$$
B(x, r)=\left\{y \in \mathbf{R}^{n}:|y-x|<r\right\}, \quad r>0 .
$$

Definition 2.2. A weight $w$ belongs to the Muckenhoupt class $\mathscr{A}_{1}$ if there exists a constant $0<A<\infty$ such that the inequality

$$
M w(x) \leq A w(x)
$$

holds for almost all $x \in \mathbf{R}^{n}$. The Muckenhoupt class $\mathscr{A}_{\infty}$ is given by

$$
\mathscr{A}_{\infty}=\bigcup_{p>1} \mathscr{A}_{p}
$$

Since the pioneering work of Muckenhoupt [24, 25, 26], these classes of weight functions have been studied in great detail, we refer, in particular, to the monographs [14], [34, Ch. V], [35], and [36, Ch. IX] for a complete account on the theory of Muckenhoupt weights. Let us mention the important feature of decomposition of $\mathscr{A}_{p}$ weights into $\mathscr{A}_{1}$ weights based on the fact that for $1 \leq p<\infty$,

$$
w \in \mathscr{A}_{p} \quad \text { if and only if there are } w_{1}, w_{2} \in \mathscr{A}_{1} \text { with } w=w_{1} w_{2}^{1-p} .
$$

Moreover, it is known that the minimum, maximum, and the sum of finitely many $\mathscr{A}_{1}$ weights yields again an $\mathscr{A}_{1}$ weight. We refer to the above-mentioned literature for proofs and further details. As usual, we use the abbreviation

$$
w(\Omega)=\int_{\Omega} w(x) d x
$$

where $\Omega \subset \mathbf{R}^{n}$ is some bounded, measurable set. Then a positive, locally integrable function $w$ on $\mathbf{R}^{n}$ belongs to $\mathscr{A}_{p}, 1 \leq p<\infty$ if and only if

$$
\frac{1}{|B|} \int_{B} f(y) d y \leq\left(\frac{c}{w(B)} \int_{B} f^{p}(x) w(x) d x\right)^{1 / p}
$$


holds for all nonnegative $f$ and all balls $B$. In particular, with $E \subset B$ and $f=\chi_{E}$, this implies that

$$
\frac{|E|}{|B|} \leq c^{\prime}\left(\frac{w(E)}{w(B)}\right)^{1 / p}, \quad E \subset B
$$

whenever $w \in \mathscr{A}_{p}, 1 \leq p<\infty$. Another property of Muckenhoupt weights that will be used in the sequel is that $w \in \mathscr{A}_{p}$ implies the existence of some number $r<p$ such that $w \in \mathscr{A}_{r}$. This is closely connected with the so-called 'reverse Hölder inequality', see [34, Ch. V, Prop. 3, Cor.]. In our case this fact will re-emerge in the number

$$
r_{w}:=\inf \left\{r \geq 1: w \in \mathscr{A}_{r}\right\}, \quad w \in \mathscr{A}_{\infty},
$$

that plays an essential role later on. Obviously, $1 \leq r_{w}<\infty$, and $w \in \mathscr{A}_{r_{w}}$ implies $r_{w}=1$.

Examples 2.3. One of the most prominent examples of a Muckenhoupt weight $w \in \mathscr{A}_{p}, 1 \leq p<\infty$, is given by $w(x)=|x|^{\varrho}$, where

$$
w(x)=|x|^{\varrho} \in \mathscr{A}_{p}
$$

if and only if

$$
\begin{cases}-n<\varrho<n(p-1) & \text { if } 1<p<\infty \\ -n<\varrho \leq 0 & \text { if } p=1\end{cases}
$$

Thus $r_{w}=1+\frac{\varrho_{+}}{n}$ and $w \in \mathscr{A}_{r_{w}}$ if $\varrho \leq 0$, whereas $w \notin \mathscr{A}_{r_{w}}$ for $\varrho>0$. We modified this example in [17] by

$$
w_{\alpha, \beta}(x)=\left\{\begin{array}{ll}
|x|^{\alpha} & \text { if } \quad|x| \leq 1, \\
|x|^{\beta} & \text { if } \quad|x|>1,
\end{array} \quad \text { with } \quad \alpha>-n, \quad \beta>-n,\right.
$$

which belongs to $\mathscr{A}_{p}, 1<p<\infty$, if and only if $-n<\alpha, \beta<n(p-1)$, and to $\mathscr{A}_{1}$ if $-n<\alpha, \beta \leq 0$. Note that $r_{w_{\alpha, \beta}}=1+\frac{\max (\alpha, \beta, 0)}{n}$.

For later use we recall a 'fractal' example studied in [16]. Let $\Gamma \subset \mathbf{R}^{n}$ be a $d$-set, $0<d<n$, in the sense of [39, Def. 3.1], [20] (which is different from [11]), i.e., there exists a Borel measure $\mu$ in $\mathbf{R}^{n}$ such that supp $\mu=\Gamma$ and there are constants $c_{1}, c_{2}>0$ such that for arbitrary $\gamma \in \Gamma$ and all $0<r<1$ holds

$$
c_{1} r^{d} \leq \mu(B(\gamma, r) \cap \Gamma) \leq c_{2} r^{d} .
$$

We proved in [16] that the weight $w_{\varkappa, \Gamma}$, given by

$$
w_{\varkappa, \Gamma}(x)= \begin{cases}\operatorname{dist}(x, \Gamma)^{\varkappa}, & \operatorname{dist}(x, \Gamma) \leq 1 \\ 1, & \operatorname{dist}(x, \Gamma) \geq 1\end{cases}
$$

satisfies

$$
w_{\varkappa, \Gamma} \in \mathscr{A}_{p} \quad \text { if and only if }-(n-d)<\varkappa<(n-d)(p-1), \quad 1<p<\infty .
$$

Consequently, $r_{w_{\varkappa, \Gamma}}=1+\frac{\max (\varkappa, 0)}{n-d}$. For further examples we refer to $[12,17,18]$. 
2.2. Function spaces of type $B_{p, q}^{s}\left(\mathbf{R}^{n}, w\right)$ and $F_{p, q}^{s}\left(\mathbf{R}^{n}, w\right)$ with $w \in \mathscr{A}_{\infty}$. Let $w \in \mathscr{A}_{\infty}$ be a Muckenhoupt weight and $0<p<\infty$. Then the weighted Lebesgue space $L_{p}\left(\mathbf{R}^{n}, w\right)$ contains all measurable functions such that

$$
\left\|f \mid L_{p}\left(\mathbf{R}^{n}, w\right)\right\|=\left(\int_{\mathbf{R}^{n}}|f(x)|^{p} w(x) d x\right)^{1 / p}
$$

is finite. Note that for $p=\infty$ one obtains the classical (unweighted) Lebesgue space,

$$
L_{\infty}\left(\mathbf{R}^{n}, w\right)=L_{\infty}\left(\mathbf{R}^{n}\right), \quad w \in \mathscr{A}_{\infty} .
$$

We thus mainly restrict ourselves to $p<\infty$ in what follows.

The Schwartz space $\mathscr{S}\left(\mathbf{R}^{n}\right)$ and its dual $\mathscr{S}^{\prime}\left(\mathbf{R}^{n}\right)$ of all complex-valued tempered distributions have their usual meaning here. Let $\varphi_{0}=\varphi \in \mathscr{S}\left(\mathbf{R}^{n}\right)$ be such that

$$
\operatorname{supp} \varphi \subset\left\{y \in \mathbf{R}^{n}:|y|<2\right\} \quad \text { and } \varphi(x)=1 \quad \text { if } \quad|x| \leq 1 \text {, }
$$

and for each $j \in \mathbf{N}$ let $\varphi_{j}(x)=\varphi\left(2^{-j} x\right)-\varphi\left(2^{-j+1} x\right)$. Then $\left\{\varphi_{j}\right\}_{j=0}^{\infty}$ forms a smooth dyadic resolution of unity. Given any $f \in \mathscr{S}^{\prime}\left(\mathbf{R}^{n}\right)$, we denote by $\mathscr{F} f$ and $\mathscr{F}^{-1} f$ its Fourier transform and its inverse Fourier transform, respectively. Let $f \in \mathscr{S}^{\prime}\left(\mathbf{R}^{n}\right)$, then the Paley-Wiener-Schwartz theorem implies that $\mathscr{F}^{-1}\left(\varphi_{j} \mathscr{F} f\right)$ is an entire analytic function on $\mathbf{R}^{n}$.

Definition 2.4. Let $0<q \leq \infty, 0<p<\infty, s \in \mathbf{R}$ and $\left\{\varphi_{j}\right\}_{j}$ a smooth dyadic resolution of unity. Assume $w \in \mathscr{A}_{\infty}$.

(i) The weighted Besov space $B_{p, q}^{s}\left(\mathbf{R}^{n}, w\right)$ is the set of all distributions $f \in \mathscr{S}^{\prime}\left(\mathbf{R}^{n}\right)$ such that

$$
\left\|f \mid B_{p, q}^{s}\left(\mathbf{R}^{n}, w\right)\right\|=\left(\sum_{j=0}^{\infty} 2^{j s q}\left\|\mathscr{F}^{-1}\left(\varphi_{j} \mathscr{F} f\right) \mid L_{p}\left(\mathbf{R}^{n}, w\right)\right\|^{q}\right)^{1 / q}
$$

is finite. In the limiting case $q=\infty$ the usual modification is required.

(ii) The weighted Triebel-Lizorkin space $F_{p, q}^{s}\left(\mathbf{R}^{n}, w\right)$ is the set of all distributions $f \in \mathscr{S}^{\prime}\left(\mathbf{R}^{n}\right)$ such that

$$
\left\|f\left|F_{p, q}^{s}\left(\mathbf{R}^{n}, w\right)\|=\|\left(\sum_{j=0}^{\infty} 2^{j s q}\left|\mathscr{F}^{-1}\left(\varphi_{j} \mathscr{F} f\right)(\cdot)\right|^{q}\right)^{1 / q}\right| L_{p}\left(\mathbf{R}^{n}, w\right)\right\|
$$

is finite. In the limiting case $q=\infty$ the usual modification is required.

Remark 2.5. The spaces $B_{p, q}^{s}\left(\mathbf{R}^{n}, w\right)$ and $F_{p, q}^{s}\left(\mathbf{R}^{n}, w\right)$ are independent of the particular choice of the smooth dyadic resolution of unity $\left\{\varphi_{j}\right\}_{j}$ appearing in their definitions. They are quasi-Banach spaces (Banach spaces for $p, q \geq 1$ ), and $\mathscr{S}\left(\mathbf{R}^{n}\right) \hookrightarrow$ $B_{p, q}^{s}\left(\mathbf{R}^{n}, w\right) \hookrightarrow \mathscr{S}^{\prime}\left(\mathbf{R}^{n}\right)$, where the first embedding is dense if $q<\infty$, similarly for the $F$-case; cf. [3]. Moreover, for $w_{0} \equiv 1 \in \mathscr{A}_{\infty}$ we re-obtain the usual (unweighted) Besov and Triebel-Lizorkin spaces; we refer, in particular, to the series of monographs by Triebel, [37, 38, 39, 40] for a comprehensive treatment of the unweighted spaces.

The above spaces with weights of type $w \in \mathscr{A}_{\infty}$ have been studied systematically by Bui first in $[3,4]$, with subsequent papers $[5,6]$. It turned out that many of the results from the unweighted situation have weighted counterparts: e.g., we have $F_{p, 2}^{0}\left(\mathbf{R}^{n}, w\right)=h_{p}\left(\mathbf{R}^{n}, w\right), 0<p<\infty$, where the latter are Hardy spaces, see [3, Thm. 1.4], and, in particular, $h_{p}\left(\mathbf{R}^{n}, w\right)=L_{p}\left(\mathbf{R}^{n}, w\right)=F_{p, 2}^{0}\left(\mathbf{R}^{n}, w\right), 1<p<\infty, w \in \mathscr{A}_{p}$, 
see [35, Ch. VI, Thm. 1]. Concerning (classical) Sobolev spaces $W_{p}^{k}\left(\mathbf{R}^{n}, w\right)$ (built upon $L_{p}\left(\mathbf{R}^{n}, w\right)$ in the usual way) it holds

$$
W_{p}^{k}\left(\mathbf{R}^{n}, w\right)=F_{p, 2}^{k}\left(\mathbf{R}^{n}, w\right), \quad k \in \mathbf{N}_{0}, \quad 1<p<\infty, \quad w \in \mathscr{A}_{p}
$$

cf. [3, Thm. 2.8]. Further details can be found in [3, 4, 14, 30].

Rychkov extended in [31] the above class of weights in order to incorporate locally regular weights, too, creating in that way the class $\mathscr{A}_{p}^{\text {loc }}$. Recent works are due to Roudenko [29, 30, 13], and Bownik [1, 2]. We partly rely on our approaches in [16, 17].

We shall later deal with function spaces of the above type on some domain $\Omega \subset \mathbf{R}^{n}$. We define these spaces by restriction, i.e.,

$$
B_{p, q}^{s}(\Omega, w)=\left\{f \in \mathscr{D}^{\prime}(\Omega): \exists g \in B_{p, q}^{s}\left(\mathbf{R}^{n}, w\right),\left.g\right|_{\Omega}=f\right\}
$$

normed with

$$
\left\|f \mid B_{p, q}^{s}(\Omega, w)\right\|=\inf \left\{\left\|g\left|B_{p, q}^{s}\left(\mathbf{R}^{n}, w\right) \|: g \in B_{p, q}^{s}\left(\mathbf{R}^{n}, w\right), g\right|_{\Omega}=f\right\},\right.
$$

similarly for $F$-spaces. In case of $w \equiv 1$ we have $B_{p, q}^{s}(\Omega, w)=B_{p, q}^{s}(\Omega)$.

Finally, we briefly describe the wavelet characterizations of Besov spaces with $\mathscr{A}_{\infty}$ weights proved in [17]. Let for $m \in \mathbf{Z}^{n}$ and $\nu \in \mathbf{N}_{0}, Q_{\nu, m}$ denote the $n$-dimensional cube with sides parallel to the axes of coordinates, centered at $2^{-\nu} m$ and with side length $2^{-\nu}$. For $0<p<\infty, \nu \in \mathbf{N}_{0}$ and $m \in \mathbf{Z}^{n}$ we denote by $\chi_{\nu, m}^{(p)}$ the $p$-normalized characteristic function of the cube $Q_{\nu, m}$,

$$
\chi_{\nu, m}^{(p)}(x)=2^{\frac{\nu n}{p}} \chi_{\nu, m}(x)= \begin{cases}2^{\frac{\nu n}{p}} & \text { for } x \in Q_{\nu, m}, \\ 0 & \text { for } x \notin Q_{\nu, m}\end{cases}
$$

hence $\left\|\chi_{\nu, m}^{(p)} \mid L_{p}\left(\mathbf{R}^{n}\right)\right\|=1$. Apart from function spaces with weights we introduce sequence spaces with weights. For $0<p<\infty, 0<q \leq \infty, \sigma \in \mathbf{R}$, and $w \in \mathscr{A}_{\infty}$, let

$$
\begin{aligned}
b_{p, q}^{\sigma}(w):= & \left\{\lambda=\left\{\lambda_{\nu, m}\right\}_{\nu, m}: \lambda_{\nu, m} \in \mathbf{C},\right. \\
& \left.\left\|\lambda\left|b_{p, q}^{\sigma}(w)\|=\|\left\{2^{\nu \sigma}\left\|\sum_{m \in \mathbf{Z}^{n}} \lambda_{\nu, m} \chi_{\nu, m}^{(p)} \mid L_{p}\left(\mathbf{R}^{n}, w\right)\right\|\right\}_{\nu \in \mathbf{N}_{0}}\right| \ell_{q}\right\|<\infty\right\}
\end{aligned}
$$

and

$$
\ell_{p}(w):=\left\{\lambda=\left\{\lambda_{m}\right\}_{m}: \lambda_{m} \in \mathbf{C},\left\|\lambda\left|\ell_{p}(w)\|=\| \sum_{m \in \mathbf{Z}^{n}} \lambda_{m} \chi_{0, m}^{(p)}\right| L_{p}\left(\mathbf{R}^{n}, w\right)\right\|<\infty\right\} .
$$

Let $\widetilde{\phi}$ be an orthogonal scaling function on $\mathbf{R}$ with compact support and of sufficiently high regularity. Let $\widetilde{\psi}$ be an associated wavelet. Then the tensor-product ansatz yields a scaling function $\phi$ and associated wavelets $\psi_{1}, \ldots, \psi_{2^{n}-1}$, all defined now on $\mathbf{R}^{n}$. We suppose $\widetilde{\phi} \in C^{N_{1}}(\mathbf{R})$ and supp $\widetilde{\phi} \subset\left[-N_{2}, N_{2}\right]$ for certain natural numbers $N_{1}$ and $N_{2}$. This implies

$$
\phi, \psi_{i} \in C^{N_{1}}\left(\mathbf{R}^{n}\right) \text { and } \operatorname{supp} \phi, \operatorname{supp} \psi_{i} \subset\left[-N_{3}, N_{3}\right]^{n},
$$

for $i=1, \ldots, 2^{n}-1$. We use the standard abbreviations

$$
\phi_{\nu, m}(x)=2^{\nu n / 2} \phi\left(2^{\nu} x-m\right) \quad \text { and } \quad \psi_{i, \nu, m}(x)=2^{\nu n / 2} \psi_{i}\left(2^{\nu} x-m\right) .
$$


For smooth weights and compactly supported wavelets it makes sense to consider the Fourier-wavelet coefficients of tempered distributions $f \in \mathscr{S}^{\prime}\left(\mathbf{R}^{n}\right)$ with respect to such an orthonormal basis. In [17, Thm. 1.13] we proved the following result.

Theorem 2.6. Let $0<p, q \leq \infty$ and let $s \in \mathbf{R}$. Let $\phi$ be a scaling function and let $\psi_{i}, i=1, \ldots, 2^{n}-1$, be the corresponding wavelets satisfying (2.20). We assume that $|s|<N_{1}$. Then a distribution $f \in \mathscr{S}^{\prime}\left(\mathbf{R}^{n}\right)$ belongs to $B_{p, q}^{s}\left(\mathbf{R}^{n}, w\right)$ if and only if $\left\|f\left|B_{p, q}^{s}\left(\mathbf{R}^{n}, w\right)\left\|^{\star}=\right\|\left\{\left\langle f, \phi_{0, m}\right\rangle\right\}_{m \in \mathbf{Z}^{n}}\right| \ell_{p}(w)\right\|+\sum_{i=1}^{2^{n}-1}\left\|\left\{\left\langle f, \psi_{i, \nu, m}\right\rangle\right\}_{\nu \in \mathbf{N}_{0}, m \in \mathbf{Z}^{n}} \mid b_{p, q}^{\sigma}(w)\right\|$

is finite, where $\sigma=s+\frac{n}{2}-\frac{n}{p}$. Furthermore, $\left\|f \mid B_{p, q}^{s}\left(\mathbf{R}^{n}, w\right)\right\|^{\star}$ may be used as an equivalent (quasi-)norm in $B_{p, q}^{s}\left(\mathbf{R}^{n}, w\right)$.

We adopt the nowadays usual custom to write $A_{p, q}^{s}$ instead of $B_{p, q}^{s}$ or $F_{p, q}^{s}$, respectively, when both scales of spaces are meant simultaneously in some context.

\section{Continuity and compactness of embeddings}

We collect some embedding results for weighted spaces that will be used later. Essentially we are interested in situations where either only the source space is weighted and the target space unweighted, or where both source and target space are weighted with the same weight. The considerations of these model cases are preceded by some general results on embeddings with possibly different weights.

3.1. General setting. We begin with a criterion obtained in [17].

Proposition 3.1. Let $w_{1}$ and $w_{2}$ be two $\mathscr{A}_{\infty}$ weights and let $-\infty<s_{2} \leq s_{1}<\infty$, $0<p_{1}, p_{2} \leq \infty, 0<q_{1}, q_{2} \leq \infty$. We put

$$
\frac{1}{p^{*}}:=\left(\frac{1}{p_{2}}-\frac{1}{p_{1}}\right)_{+} \text {and } \frac{1}{q^{*}}:=\left(\frac{1}{q_{2}}-\frac{1}{q_{1}}\right)_{+} .
$$

(i) There is a continuous embedding $B_{p_{1}, q_{1}}^{s_{1}}\left(\mathbf{R}^{n}, w_{1}\right) \hookrightarrow B_{p_{2}, q_{2}}^{s_{2}}\left(\mathbf{R}^{n}, w_{2}\right)$ if and only if

$$
\left\{2^{-\nu\left(s_{1}-s_{2}\right)}\left\|\left\{\left(w_{2}\left(Q_{\nu, m}\right)\right)^{1 / p_{2}}\left(w_{1}\left(Q_{\nu, m}\right)\right)^{-1 / p_{1}}\right\}_{m} \mid \ell_{p *}\right\|\right\}_{\nu} \in \ell_{q^{*}} .
$$

(ii) The embedding $B_{p_{1}, q_{1}}^{s_{1}}\left(\mathbf{R}^{n}, w_{1}\right) \hookrightarrow B_{p_{2}, q_{2}}^{s_{2}}\left(\mathbf{R}^{n}, w_{2}\right)$ is compact if and only if (3.2) holds and, in addition,

$$
\lim _{\nu \rightarrow \infty} 2^{-\nu\left(s_{1}-s_{2}\right)}\left\|\left\{\left(w_{2}\left(Q_{\nu, m}\right)\right)^{1 / p_{2}}\left(w_{1}\left(Q_{\nu, m}\right)\right)^{-1 / p_{1}}\right\}_{m} \mid \ell_{p *}\right\|=0 \quad \text { if } q^{*}=\infty,
$$

and

$$
\lim _{|m| \rightarrow \infty}\left(w_{2}\left(Q_{\nu, m}\right)\right)^{-1 / p_{2}}\left(w_{1}\left(Q_{\nu, m}\right)\right)^{1 / p_{1}}=\infty \quad \text { for all } \nu \in \mathbf{N}_{0} \quad \text { if } p^{*}=\infty .
$$

Remark 3.2. The result is proved in [17, Prop. 2.1] based on the wavelet decomposition Theorem 2.6, the commutative diagram

$$
\begin{array}{ccc}
B_{p_{1}, q_{1}}^{s_{1}}\left(\mathbf{R}^{n}, w_{1}\right) & \frac{T}{T^{-1}} & b_{p_{1}, q_{1}}^{\sigma_{1}}\left(w_{1}\right) \\
\text { Id } \downarrow & & \downarrow \text { id } \\
B_{p_{2}, q_{2}}^{s_{2}}\left(\mathbf{R}^{n}, w_{2}\right) & \frac{S}{S^{-1}} & b_{p_{2}, q_{2}}^{\sigma_{2}}\left(w_{2}\right)
\end{array}
$$


with appropriate isomorphisms $S$ and $T$ and the general result [22, Thm. 1]. Similarly, with an appropriate isomorphism $A$ one can reduce the investigation of the embeddings of two weighted sequence spaces to the study of embeddings of a weighted space into an unweighted one, using

$$
\begin{aligned}
& b_{p_{1}, q_{1}}^{\sigma_{1}}\left(w_{1}\right) \stackrel{A}{\underset{A^{-1}}{\rightleftharpoons}} b_{p_{1}, q_{1}}^{\sigma_{1}}\left(\frac{w_{1}}{w_{2}}\right) \\
& \text { Id } \downarrow \text { id } \\
& b_{p_{2}, q_{2}}^{\sigma_{2}}\left(w_{2}\right) \stackrel{A^{-1}}{A} \quad b_{p_{2}, q_{2}}^{\sigma_{2}}
\end{aligned}
$$

Remark 3.3. In view of (2.12) it is clear that we obtain unweighted Besov spaces if $p_{1}=p_{2}=\infty$. Then by $(2.6), w_{1}\left(Q_{\nu, m}\right)=w_{2}\left(Q_{\nu, m}\right)=2^{-\nu n}$ for all $\nu \in \mathbf{N}_{0}$ and $m \in \mathbf{Z}^{n}$, such that (3.2) leads to $p^{*}=\infty$, i.e., $p_{1} \leq p_{2}$, and

$$
\delta:=s_{1}-\frac{n}{p_{1}}-s_{2}+\frac{n}{p_{2}}>0,
$$

with the extension to $\delta=0$ if $q_{1} \leq q_{2}$, i.e., $q^{*}=\infty$. Moreover, by (3.4), the embedding is never compact (as is well-known in this case).

As already mentioned, we might restrict ourselves to the situation when only the source space is weighted, and the target space unweighted,

$$
\operatorname{id}_{w}: B_{p_{1}, q_{1}}^{s_{1}}\left(\mathbf{R}^{n}, w\right) \hookrightarrow B_{p_{2}, q_{2}}^{s_{2}}\left(\mathbf{R}^{n}\right),
$$

where $w \in \mathscr{A}_{\infty}$. Moreover, we shall assume in the sequel that $p_{1}<\infty$ for convenience, as otherwise we have $B_{p_{1}, q_{1}}^{s_{1}}\left(\mathbf{R}^{n}, w\right)=B_{p_{1}, q_{1}}^{s_{1}}\left(\mathbf{R}^{n}\right)$, recall $(2.12)$, and we arrive at the unweighted situation in (3.6) which is well-known already. Then we stick to the general assumptions

$$
-\infty<s_{2} \leq s_{1}<\infty, \quad 0<p_{1}<\infty, \quad 0<p_{2} \leq \infty, \quad 0<q_{1}, q_{2} \leq \infty .
$$

Occasionally we will formulate some results in the 'double-sided' weighted situation, in particular, corresponding to the setting

$$
\operatorname{id}_{w w}: B_{p_{1}, q_{1}}^{s_{1}}\left(\mathbf{R}^{n}, w\right) \hookrightarrow B_{p_{2}, q_{2}}^{s_{2}}\left(\mathbf{R}^{n}, w\right),
$$

with

$$
-\infty<s_{2} \leq s_{1}<\infty, \quad 0<p_{1}, p_{2}<\infty, \quad 0<q_{1}, q_{2} \leq \infty .
$$

Before we collect and extend our results from [17] according to (3.6) and (3.8), we insert some short preparation in view of Proposition 3.1. We have to consider expressions of type $w_{2}\left(Q_{\nu, m}\right)^{1 / p_{2}} w_{1}\left(Q_{\nu, m}\right)^{-1 / p_{1}}$, i.e., $2^{-\nu n / p_{2}} w\left(Q_{\nu, m}\right)^{-1 / p_{1}}$ in case of (3.6), and $w\left(Q_{\nu, m}\right)^{\frac{1}{p_{2}}-\frac{1}{p_{1}}}$ in case of (3.8). Let $w \in \mathscr{A}_{r}, 1 \leq r<\infty$, then by (2.7),

$$
w\left(Q_{\nu, m}\right) \geq c 2^{-\nu n r} w\left(Q_{0, l}\right) \quad \text { for all } Q_{\nu, m} \subset Q_{0, l}, \quad \nu \in \mathbf{N}_{0}, m, l \in \mathbf{Z}^{n},
$$

such that for any $\varkappa<0, w\left(Q_{\nu, m}\right)^{\varkappa} \leq c^{\prime} 2^{-\nu n r \varkappa} w\left(Q_{0, l}\right)^{\varkappa}$, where $Q_{\nu, m} \subset Q_{0, l}$, and thus

$$
\left\|\left\{w\left(Q_{\nu, m}\right)^{\varkappa}\right\}_{m} \mid \ell_{\infty}\right\| \leq c 2^{-\nu r n \varkappa}\left(\inf _{l} w\left(Q_{0, l}\right)\right)^{\varkappa}, \quad \varkappa<0, \nu \in \mathbf{N}_{0} .
$$

Moreover, for arbitrary $\gamma>0$, (3.10) leads to

$$
\lim _{|m| \rightarrow \infty} w\left(Q_{\nu, m}\right)^{\gamma}=\infty \text { for all } \nu \in \mathbf{N}_{0} \text { if and only if } \lim _{|l| \rightarrow \infty} w\left(Q_{0, l}\right)=\infty
$$


Summarizing the above considerations, we find that for embeddings of type (3.6) or (3.8) the conditions

$$
\inf _{l} w\left(Q_{0, l}\right) \geq c>0
$$

and

$$
\lim _{|l| \rightarrow \infty} w\left(Q_{0, l}\right)=\infty
$$

are essential when $p_{1} \leq p_{2}$, that is, $p^{*}=\infty$. If $p^{*}<\infty$, then careful calculation leads to

$$
\left\|\left\{w\left(Q_{\nu, m}\right)^{\varkappa}\right\}_{m}\left|\ell_{p^{*}}\left\|\leq c 2^{-\nu r n \varkappa+\nu \frac{n}{p^{*}}}\right\|\left\{w\left(Q_{0, l}\right)^{\varkappa}\right\}_{l}\right| \ell_{p^{*}}\right\|, \quad \varkappa<0, \nu \in \mathbf{N}_{0},
$$

as the counterpart of (3.11). Therefore, the adequate extension of (3.12) to $p^{*}<\infty$ reads as

$$
\left\|\left\{w\left(Q_{0, l}\right)^{-1 / p_{1}}\right\}_{l} \mid \ell_{p^{*}}\right\| \leq c<\infty .
$$

3.2. Embeddings in one-sided weighted situations. We begin with a special case studied in [17].

Example 3.4. For later use we recall the corresponding result for a weight of type (2.9) with $\alpha, \beta>-n$. Let the parameters be given by (3.7). In [17, Prop. 2.6] we proved the following:

(i) The embedding $B_{p_{1}, q_{1}}^{s_{1}}\left(\mathbf{R}^{n}, w_{\alpha, \beta}\right) \hookrightarrow B_{p_{2}, q_{2}}^{s_{2}}\left(\mathbf{R}^{n}\right)$ is continuous if and only if

$$
\begin{cases}\text { either } & \beta \geq 0 \text { if } p^{*}=\infty, \\ \text { or } & \frac{\beta}{p_{1}}>\frac{n}{p^{*}} \text { if } p^{*}<\infty,\end{cases}
$$

and one of the following conditions is satisfied,

$$
\begin{aligned}
& \delta \geq \max \left(\frac{\alpha}{p_{1}}, \frac{n}{p^{*}}\right) \quad \text { if } q^{*}=\infty, \text { with } \frac{n}{p^{*}} \neq \frac{\alpha}{p_{1}} \text { if } p^{*}<\infty, \\
& \delta>\max \left(\frac{\alpha}{p_{1}}, \frac{n}{p^{*}}\right) \quad \text { otherwise. }
\end{aligned}
$$

(ii) The embedding $A_{p_{1}, q_{1}}^{s_{1}}\left(\mathbf{R}^{n}, w_{\alpha, \beta}\right) \hookrightarrow A_{p_{2}, q_{2}}^{s_{2}}\left(\mathbf{R}^{n}\right)$ is compact if and only if

$$
\frac{\beta}{p_{1}}>\frac{n}{p^{*}} \text { and } \delta>\max \left(\frac{n}{p^{*}}, \frac{\alpha}{p_{1}}\right) \text {. }
$$

We now briefly summarize our results [17, Cors. 2.11, 2.13].

Corollary 3.5. Let the parameters be given by (3.7), and $w \in \mathscr{A}_{\infty}$ with $r_{w}$ given by (2.8).

(i) Let

$$
\delta>\frac{n}{p^{*}}+\frac{n}{p_{1}}\left(r_{w}-1\right) .
$$

If $p_{1} \leq p_{2}$, then $\mathrm{id}_{w}$ in (3.6) is continuous if and only if (3.14) is satisfied. It is compact if and only if (3.13) holds.

If $p_{1}>p_{2}$, then $\mathrm{id}_{w}$ in (3.6) is compact if and only if (3.14) holds.

(ii) Let $\delta<\frac{n}{p^{*}}$, then $B_{p_{1}, q_{1}}^{s_{1}}\left(\mathbf{R}^{n}, w\right)$ is not embedded in $B_{p_{2}, q_{2}}^{s_{2}}\left(\mathbf{R}^{n}\right)$. 
(iii) Let $\delta=\frac{n}{p^{*}}$. If $q^{*}<\infty$, then $B_{p_{1}, q_{1}}^{s_{1}}\left(\mathbf{R}^{n}, w\right)$ is not embedded in $B_{p_{2}, q_{2}}^{s_{2}}\left(\mathbf{R}^{n}\right)$. If $q^{*}=\infty$ and $w \in \mathscr{A}_{r_{w}}$, that is, $r_{w}=1$, then $\mathrm{id}_{w}$ in (3.6) is continuous if and only if (3.14) is satisfied.

(iv) If $\frac{n}{p^{*}}<\delta<\frac{n}{p^{*}}+\frac{n}{p_{1}}\left(r_{w}-1\right)$, then for every $r>r_{w}$ there exists an $\mathscr{A}_{r}$ weight $v$ satisfying (3.14) such that the space $B_{p_{1}, q_{1}}^{s_{1}}\left(\mathbf{R}^{n}, v\right)$ is not embedded in $B_{p_{2}, q_{2}}^{s_{2}}\left(\mathbf{R}^{n}\right)$.

Remark 3.6. Apart from the case $w \in \mathscr{A}_{1}$, see also Corollary 3.7 below, there remains the gap

$$
\frac{n}{p^{*}}<\delta \leq \frac{n}{p^{*}}+\frac{n}{p_{1}}\left(r_{w}-1\right)
$$

where we only have the complementing assertion (iv). However, it is not surprising that general features of $w$ like $r_{w}$ and (3.14) are not appropriately adapted for the interplay with the parameters (3.7) as required in Proposition 3.1. Reviewing, for instance, Example 3.4 one realizes that more information of the weight is used than reflected by $r_{w}$ and (3.14) only. For example, Corollary 3.5 covers only the cases $\beta \geq 0$ and $\delta>\frac{\max (\alpha, \beta)}{p_{1}}$ in Example 3.4 (with $p_{1} \leq p_{2}$ ), thus neglecting the (admitted) situations when $\frac{\max (\alpha, 0)}{p_{1}} \leq \delta \leq \frac{\max (\alpha, \beta)}{p_{1}}$. But this requires further information on the weight, as already mentioned.

Before we come to the double-sided weighted situation we want to clarify the situation for $\mathscr{A}_{1}$ weights, for which $r_{w}=1$.

Corollary 3.7. Let the parameters be given by (3.7) and let $w \in \mathscr{A}_{1}$ such that $r_{w}=1$.

(i) Then $\mathrm{id}_{w}$ in (3.6) is continuous if and only if the following three conditions hold,

$$
\begin{aligned}
& \text { a) } \quad \inf _{l} w\left(Q_{0, l}\right) \geq c>0 \\
& \text { b) }\left\{\begin{array}{lll}
\delta>0 & \text { if } \quad q^{*}<\infty \\
\delta \geq 0 & \text { if } \quad q^{*}=\infty
\end{array}\right. \\
& \text { c) } p_{1} \leq p_{2}
\end{aligned}
$$

Otherwise $B_{p_{1}, q_{1}}^{s_{1}}\left(\mathbf{R}^{n}, w\right)$ is not embedded in $B_{p_{2}, q_{2}}^{s_{2}}\left(\mathbf{R}^{n}\right)$.

(ii) The embedding $\mathrm{id}_{w}$ in (3.6) is never compact.

Proof. In view of Corollary 3.5 it is sufficient to prove that for $w \in \mathscr{A}_{1}$ conditions (3.13) and (3.14) are never satisfied, that is, we only have to disprove (3.13) in that case. So assume that $w \in \mathscr{A}_{\infty}$ and (3.13) holds. Then there is some number $k_{0} \in \mathbf{Z}^{n}$ such that

$$
\inf _{l \in \mathbf{Z}^{n}} w\left(Q_{0, l}\right)=w\left(Q_{0, k_{0}}\right)>0 .
$$

On the other hand, (3.13) implies for arbitrary $N \in \mathbf{N}$ the existence of some number $L=L(N) \in \mathbf{N}$ such that $w\left(Q_{0, l}\right)>N w\left(Q_{0, k_{0}}\right)$ for all $l \in \mathbf{Z}^{n},|l|>L(N)$. Let $\Lambda_{N}=\left\{m \in \mathbf{Z}^{n}:|m| \leq L(N)\right\} \subset \mathbf{Z}^{n}$, that is, we have

$$
w\left(Q_{0, l}\right)>N w\left(Q_{0, k_{0}}\right) \quad \text { for all } l \notin \Lambda_{N} .
$$

Clearly, $k_{0} \in \Lambda_{N}$, hence $\# \Lambda_{N} \geq 1$. Let $\Gamma_{N} \supset \Lambda_{N}$ be the index set of a collection of cubes that contains twice the number of cubes as for $\Lambda_{N}$, that is, $\#\left(\Gamma_{N} \backslash \Lambda_{N}\right)=\# \Lambda_{N}$. 
We set

$$
B_{N}=\bigcup_{m \in \Gamma_{N}} Q_{0, m} \supset \bigcup_{l \in \Lambda_{N}} Q_{0, l}, \quad\left|B_{N}\right|=\sum_{m \in \Gamma_{N}}\left|Q_{0, m}\right|=2 \# \Lambda_{N} .
$$

This leads to

$$
\begin{aligned}
\frac{w\left(B_{N}\right)}{\left|B_{N}\right|} & =\frac{\sum_{m \in \Lambda_{N}} w\left(Q_{0, m}\right)+\sum_{m \in \Gamma_{N} \backslash \Lambda_{N}} w\left(Q_{0, m}\right)}{2 \# \Lambda_{N}} \\
& >\frac{w\left(Q_{0, k_{0}}\right) \# \Lambda_{N}+N w\left(Q_{0, k_{0}}\right) \#\left(\Gamma_{N} \backslash \Lambda_{N}\right)}{2 \# \Lambda_{N}}=\frac{N+1}{2} w\left(Q_{0, k_{0}}\right)
\end{aligned}
$$

in view of (3.23) and (3.24). On the other hand, $k_{0} \in \Lambda_{N}$ implies $Q_{0, k_{0}} \subset B_{N}$ such that (2.7) with $p=1, E=Q_{0, k_{0}}$ and $B=B_{N}$ reads as

$$
\frac{w\left(B_{N}\right)}{\left|B_{N}\right|} \leq c^{\prime} \frac{w\left(Q_{0, k_{0}}\right)}{\left|Q_{0, k_{0}}\right|}=c^{\prime} w\left(Q_{0, k_{0}}\right)
$$

for some $c^{\prime}>0$ which contradicts (3.25) for $N \rightarrow \infty$.

Remark 3.8. We compare the above result with Example 3.4. We have $w_{\alpha, \beta} \in$ $\mathscr{A}_{1}$ if $-n<\alpha, \beta \leq 0$ by Example 2.3, hence Example 3.4(i) leads to $\alpha \leq \beta=0$, $p_{1} \leq p_{2}$ and (3.21), and Example 3.4(ii) gives (ii) above.

3.3. Embeddings in double-sided weighted situations. We turn to doublesided weighted embeddings and begin with natural counterparts of well-known unweighted situations.

Proposition 3.9. Let $0<q \leq \infty, 0<p<\infty, s \in \mathbf{R}$ and $w \in \mathscr{A}_{\infty}$.

(i) Let $-\infty<s_{1} \leq s_{0}<\infty$ and $0<q_{0} \leq q_{1} \leq \infty$, then

$$
A_{p, q}^{s_{0}}\left(\mathbf{R}^{n}, w\right) \hookrightarrow A_{p, q}^{s_{1}}\left(\mathbf{R}^{n}, w\right) \quad \text { and } \quad A_{p, q_{0}}^{s}\left(\mathbf{R}^{n}, w\right) \hookrightarrow A_{p, q_{1}}^{s}\left(\mathbf{R}^{n}, w\right) .
$$

(ii) We have

$$
B_{p, \min (p, q)}^{s}\left(\mathbf{R}^{n}, w\right) \hookrightarrow F_{p, q}^{s}\left(\mathbf{R}^{n}, w\right) \hookrightarrow B_{p, \max (p, q)}^{s}\left(\mathbf{R}^{n}, w\right) .
$$

(iii) Assume that there are numbers $c>0, d>0$ such that for all balls,

$$
w(B(x, r)) \geq c r^{d}, \quad 0<r \leq 1, \quad x \in \mathbf{R}^{n} .
$$

Let $0<p_{0}<p<p_{1}<\infty,-\infty<s_{1}<s<s_{0}<\infty$ satisfy

$$
s_{0}-\frac{d}{p_{0}}=s-\frac{d}{p}=s_{1}-\frac{d}{p_{1}} .
$$

Then

$$
B_{p_{0}, q}^{s_{0}}\left(\mathbf{R}^{n}, w\right) \hookrightarrow B_{p_{1}, q}^{s_{1}}\left(\mathbf{R}^{n}, w\right)
$$

and

$$
B_{p_{0}, p}^{s_{0}}\left(\mathbf{R}^{n}, w\right) \hookrightarrow F_{p, q}^{s}\left(\mathbf{R}^{n}, w\right) \hookrightarrow B_{p_{1}, p}^{s_{1}}\left(\mathbf{R}^{n}, w\right) .
$$

Remark 3.10. The above embeddings are natural extensions from the unweighted case $w \equiv 1$, see [37, Prop. 2.3.2/2, Thm. 2.7.1] and [32, Thm. 3.2.1]. The above result essentially coincides with [3, Thm. 2.6] and can be found in [17, Prop. 1.8]. 
We next come to situation (3.8). First note that we only have to consider the case $p_{1} \leq p_{2}$ since there cannot be a continuous embedding of type if $p_{1}>p_{2}, w \in \mathscr{A}_{\infty}$, for if (at least) (3.12) is assumed to hold, then (3.10) implies for some $r, 1 \leq r<\infty$, that

$$
w\left(Q_{\nu, m}\right) \geq c^{\prime} 2^{-\nu n r} \quad \text { for all } \nu \in \mathbf{N}_{0}, m \in \mathbf{Z}^{n}
$$

such that

$$
\left\|\left\{w\left(Q_{\nu, m}\right)^{\frac{1}{p_{2}}-\frac{1}{p_{1}}}\right\}_{m} \mid \ell_{p^{*}}\right\|=\left(\sum_{m} w\left(Q_{\nu, m}\right)\right)^{1 / p^{*}}
$$

diverges for any $\nu \in \mathbf{N}_{0}$.

Example 3.11. Let the parameters be given by (3.9). In [17, Prop. 2.8] we obtained the following counterpart of Example 3.4.

(i) The embedding $B_{p_{1}, q_{1}}^{s_{1}}\left(\mathbf{R}^{n}, w_{\alpha, \beta}\right) \hookrightarrow B_{p_{2}, q_{2}}^{s_{2}}\left(\mathbf{R}^{n}, w_{\alpha, \beta}\right)$ is continuous if and only if

$$
p_{1} \leq p_{2}, \quad \beta\left(\frac{1}{p_{1}}-\frac{1}{p_{2}}\right) \geq 0
$$

and one of the following conditions is satisfied,

$$
\begin{aligned}
& \delta \geq \max (\alpha, 0)\left(\frac{1}{p_{1}}-\frac{1}{p_{2}}\right) \quad \text { if } q^{*}=\infty, \\
& \delta>\max (\alpha, 0)\left(\frac{1}{p_{1}}-\frac{1}{p_{2}}\right) \quad \text { if } q^{*}<\infty .
\end{aligned}
$$

(ii) The embedding $A_{p_{1}, q_{1}}^{s_{1}}\left(\mathbf{R}^{n}, w_{\alpha, \beta}\right) \hookrightarrow A_{p_{2}, q_{2}}^{s_{2}}\left(\mathbf{R}^{n}, w_{\alpha, \beta}\right)$ is compact if and only if

$$
\beta>0, \quad p_{1}<p_{2} \quad \text { and } \delta>\max (\alpha, 0)\left(\frac{1}{p_{1}}-\frac{1}{p_{2}}\right) .
$$
follows.

Our general result in the double-sided weighted context [17, Cor. 2.14] reads as

Corollary 3.12. Let the parameters be given by (3.9) with $p_{1}<p_{2}$. Let $w \in \mathscr{A}_{\infty}$ and $r_{w}$ be given by (2.8).

(i) Let

$$
\delta>\left(r_{w}-1\right)\left(\frac{n}{p_{1}}-\frac{n}{p_{2}}\right) .
$$

The embedding (3.8) is continuous if and only if (3.12) is satisfied. It is compact if and only if (3.13) is satisfied.

(ii) Let $\delta<0$, then $B_{p_{1}, q_{1}}^{s_{1}}\left(\mathbf{R}^{n}, w\right)$ is not embedded into $B_{p_{2}, q_{2}}^{s_{2}}\left(\mathbf{R}^{n}, w\right)$.

(iii) Let $\delta=0$. When $q^{*}<\infty$, then $B_{p_{1}, q_{1}}^{s_{1}}\left(\mathbf{R}^{n}, w\right)$ is not embedded into $B_{p_{2}, q_{2}}^{s_{2}}\left(\mathbf{R}^{n}, w\right)$. When $q^{*}=\infty$ and $w \in \mathscr{A}_{r_{w}}$, that is, $r_{w}=1$, then $\mathrm{id}_{w w}$ in (3.8) is continuous if and only if (3.12) is satisfied.

(iv) If the condition (3.34) does not hold, then for every $r>r_{w}$ there exists an $\mathscr{A}_{r}$ weight $v$ satisfying (3.12) such that the space $B_{p_{1}, q_{1}}^{s_{1}}\left(\mathbf{R}^{n}, v\right)$ is not embedded into $B_{p_{2}, q_{2}}^{s_{2}}\left(\mathbf{R}^{n}, v\right)$. 
Remark 3.13. Note that the compactness in (i) is in some sense surprising as it is different from the unweighted situation $w \equiv 1$ (where one cannot have a compact embedding as is well-known). Of course, there is no contradiction as (3.13) is not satisfied in this case.

Moreover, Corollary 3.12 refines Proposition 3.9(iii) in some sense: Assume that (3.12) is satisfied, then since for an arbitrary ball $B(x, \varrho)$ with radius $0<\varrho<1$ there is some $m \in \mathbf{Z}^{n}$ such that $B(x, \varrho) \subset Q_{0, m}$ (apart from a universal constant) and (2.7) implies for $w \in \mathscr{A}_{r}$ that

$$
w(B(x, \varrho)) \geq c \varrho^{n r} w\left(Q_{0, m}\right),
$$

we obtain (3.27) with $d=r n$. The limiting case $\delta=(r-1)\left(\frac{n}{p_{1}}-\frac{n}{p_{2}}\right)$ in (3.34) coincides with (3.28) for $d=n r$ such that (3.29) covers the continuity of the embedding (3.8).

Again we clarify the result for $\mathscr{A}_{1}$ weights.

Corollary 3.14. Let the parameters be given by (3.7) with $p_{1}<p_{2}$ and let $w \in \mathscr{A}_{1}$ such that $r_{w}=1$.

(i) Then $\mathrm{id}_{w w}$ in (3.8) is continuous if and only if the following two conditions hold,

$$
\begin{aligned}
& \text { a) } \quad \inf _{l} w\left(Q_{0, l}\right) \geq c>0 \\
& \text { b) }\left\{\begin{array}{lll}
\delta>0 & \text { if } \quad q^{*}<\infty \\
\delta \geq 0 & \text { if } \quad q^{*}=\infty
\end{array}\right.
\end{aligned}
$$

Otherwise $B_{p_{1}, q_{1}}^{s_{1}}\left(\mathbf{R}^{n}, w\right)$ is not embedded in $B_{p_{2}, q_{2}}^{s_{2}}\left(\mathbf{R}^{n}, w\right)$.

(ii) The embedding $\mathrm{id}_{w w}$ in (3.8) is never compact.

Proof. Recall from the proof of Corollary 3.7 that $w \in \mathscr{A}_{1}$ contradicts (3.13).

In case of $p_{1}=p_{2}$ there is no direct influence of the weights on the continuity or compactness of the embedding (3.8).

Corollary 3.15. Let the parameters be given by (3.9) with $p_{1}=p_{2}$. Let $w \in$ $\mathscr{A}_{\infty}$. Then the embedding (3.8) is continuous if and only if

$$
\left\{\begin{array}{l}
s_{1}-s_{2}>0 \quad \text { if } q^{*}<\infty \\
s_{1}-s_{2} \geq 0 \quad \text { if } q^{*}=\infty
\end{array}\right.
$$

The embedding (3.8) is never compact.

Remark 3.16. Before we turn to a refined study of compact embeddings in the next section it seems worth noticing that the weight class $\mathscr{A}_{1}$ is obviously too small or weak in order to imply compactness of either the embedding $\mathrm{id}_{w}$ in (3.6) or $\mathrm{id}_{w w}$ in (3.8), recall Corollaries 3.7(ii), 3.14(ii), and 3.15. This resembles the unweighted situation, see also Remark 3.3.

\section{Singularities, entropy and approximation numbers}

We concentrate on compact embeddings now, in particular, on their entropy and approximation numbers. Let $X, Y$ be two quasi-Banach spaces and let $T: X \rightarrow Y$ be a bounded linear operator. The $k$-th (dyadic) entropy number of $T, k \in \mathbf{N}$, is defined as

$$
e_{k}(T)=\inf \left\{\varepsilon>0: T\left(B_{X}\right) \text { can be covered by } 2^{k-1} \text { balls of radius } \varepsilon \text { in } Y\right\},
$$


where $B_{X}$ denotes the closed unit ball in $X$. Due to the well known fact that

$$
T: X \rightarrow Y \text { is compact if and only if } \lim _{k \rightarrow \infty} e_{k}(T: X \rightarrow Y)=0,
$$

the entropy numbers can be viewed as a quantification of the notion of compactness. On the other hand, the $k$-th approximation number of $T$ is defined as

$$
a_{k}(T)=\inf \{\|T-L\|: \operatorname{rank} L<k\} .
$$

If $a_{k}(T) \rightarrow 0$ for $k \rightarrow \infty$, then $T$ is compact. So the asymptotic behaviour of approximation numbers also gives us the quantitative analysis of compactness of the operator. Further properties like multiplicativity and additivity, as well as applications of entropy and approximation numbers can be found in [8, 9, 10, 28].

We study the case of compact embeddings of type (3.6) or (3.8) for (general) weights $w \in \mathscr{A}_{r}, r \geq 1$, but first motivate our approach by recalling the result corresponding to Example 3.4(ii). In [17, Thms. 3.4, 4.3] we proved the following.

Example 4.1. Let the parameters satisfy (3.7) and let the weight $w \in \mathscr{A}_{\infty}$ be of type (2.9) with (3.18).

(i) If $\frac{\beta}{p_{1}} \neq \delta$, then

$$
e_{k}\left(A_{p_{1}, q_{1}}^{s_{1}}\left(\mathbf{R}^{n}, w\right) \hookrightarrow A_{p_{2}, q_{2}}^{s_{2}}\left(\mathbf{R}^{n}\right)\right) \sim k^{-\min \left(\frac{s_{1}-s_{2}}{n}, \frac{\beta}{n p_{1}}+\frac{1}{p_{1}}-\frac{1}{p_{2}}\right)}, \quad k \in \mathbf{N} .
$$

(ii) If $\frac{\beta}{p_{1}}=\delta$ and $\tau=\frac{s_{1}-s_{2}}{n}+\frac{1}{q_{2}}-\frac{1}{q_{1}} \neq 0$, then

$$
e_{k}\left(B_{p_{1}, q_{1}}^{s_{1}}\left(\mathbf{R}^{n}, w\right) \hookrightarrow B_{p_{2}, q_{2}}^{s_{2}}\left(\mathbf{R}^{n}\right)\right) \sim k^{-\frac{s_{1}-s_{2}}{n}}(1+\log k)^{\max (\tau, 0)}, \quad k \in \mathbf{N} .
$$

(iii) Let $\frac{\beta}{p_{1}} \neq \delta$, then

where

$$
a_{k}\left(A_{p_{1}, q_{1}}^{s_{1}}\left(\mathbf{R}^{n}, w\right) \hookrightarrow A_{p_{2}, q_{2}}^{s_{2}}\left(\mathbf{R}^{n}\right)\right) \sim k^{-\varkappa}, \quad k \in \mathbf{N},
$$

$$
\varkappa= \begin{cases}\frac{\min \left(\delta, \beta / p_{1}\right)}{n}+\frac{1}{p^{*}} & \text { if } 0<p_{1} \leq p_{2} \leq 2, \text { or } 2 \leq p_{1} \leq p_{2} \leq \infty, \\ \frac{\min \left(\delta, \beta / p_{1}\right)}{n}+\frac{1}{2}-\frac{1}{\min \left(p_{1}^{\prime}, p_{2}\right)} & \text { if } 0<p_{1}\left(\frac{\beta}{n}+1\right)^{-1}<p_{2}<p_{1}<\infty, \\ & \text { and } \min \left(\frac{\beta}{p_{1}}, \delta\right)>\frac{n}{\min \left(p_{1}^{\prime}, p_{2}\right)}, \\ \frac{\min \left(\delta, \beta / p_{1}\right)}{n} \cdot \frac{\min \left(p_{1}^{\prime}, p_{2}\right)}{2} & \text { if } 0<p_{1}<2<p_{2} \leq \infty \text { with }\left(p_{1}, p_{2}\right) \neq(1, \infty) \\ & \text { and } \min \left(\frac{\beta}{p_{1}}, \delta\right)<\frac{n}{\min \left(p_{1}^{\prime}, p_{2}\right)} .\end{cases}
$$

Apart from the (well-known) special phenomenon of approximation numbers to distinguish between situations where both parameters $p_{i}$ are on the same or different sides of the number 2, it is obvious that the asymptotic behaviour of entropy and approximation numbers depends upon the differential dimension $\delta$ and the singularity behaviour of $w$ characterized in the above example in terms of $\alpha$ and $\beta$, recall (2.9). More precisely, the influence of the local singularities - indicated by $\alpha$ here is restricted to condition (3.18) ensuring compactness. This observation is further strengthened by the corresponding proofs in [17]: even the entropy and approximation numbers of the local version of these embeddings (denoted by $\operatorname{Id}_{1}$ in [17]) are independent of $\alpha$ (apart from (3.18), of course).

Therefore we want to investigate this phenomenon in general and begin with a refined study of the singularity behaviour of Muckenhoupt $\mathscr{A}_{\infty}$ weights. 
4.1. Singularities. Let $w_{1}, w_{2} \in \mathscr{A}_{\infty}$. We introduce the following notion of their set of singularities $\mathbf{S}_{\text {sing }}\left(w_{1}, w_{2}\right)$.

Definition 4.2. For $w_{1}, w_{2} \in \mathscr{A}_{\infty}$ we define the set of singularities $\mathbf{S}_{\text {sing }}\left(w_{1}, w_{2}\right)$ by

$$
\mathbf{S}_{\text {sing }}\left(w_{1}, w_{2}\right)=\mathbf{S}_{0}\left(w_{1}, w_{2}\right) \cup \mathbf{S}_{\infty}\left(w_{1}, w_{2}\right),
$$

where

$$
\begin{gathered}
\mathbf{S}_{0}\left(w_{1}, w_{2}\right)=\left\{x_{0} \in \mathbf{R}^{n}: \inf _{Q_{\nu, m} \ni x_{0}} \frac{w_{1}\left(Q_{\nu, m}\right)}{w_{2}\left(Q_{\nu, m}\right)}=0\right\}, \\
\mathbf{S}_{\infty}\left(w_{1}, w_{2}\right)=\left\{x_{0} \in \mathbf{R}^{n}: \sup _{Q_{\nu, m} \ni x_{0}} \frac{w_{1}\left(Q_{\nu, m}\right)}{w_{2}\left(Q_{\nu, m}\right)}=\infty\right\} .
\end{gathered}
$$

Remark 4.3. In case of $w_{2} \equiv 1, w_{1} \equiv w \in \mathscr{A}_{\infty}$, we shall only write $\mathbf{S}_{\infty}(w)=$ $\mathbf{S}_{\infty}(w, 1)$ and $\mathbf{S}_{0}(w)=\mathbf{S}_{0}(w, 1)$, that is,

$$
\begin{aligned}
\mathbf{S}_{0}(w) & =\left\{x_{0} \in \mathbf{R}^{n}: \inf _{Q_{\nu, m} \ni x_{0}} \frac{w\left(Q_{\nu, m}\right)}{\left|Q_{\nu, m}\right|}=0\right\}, \\
\mathbf{S}_{\infty}(w) & =\left\{x_{0} \in \mathbf{R}^{n}: \sup _{Q_{\nu, m} \ni x_{0}} \frac{w\left(Q_{\nu, m}\right)}{\left|Q_{\nu, m}\right|}=\infty\right\},
\end{aligned}
$$

such that

$$
\mathbf{S}_{\text {sing }}(w)=\mathbf{S}_{0}(w) \cup \mathbf{S}_{\infty}(w) .
$$

Plainly, if $w_{1} \sim w_{2}$, i.e., there are positive numbers $c_{2}>c_{1}$ such that for all $x \in \mathbf{R}^{n}$, $c_{1} w_{2}(x) \leq w_{1}(x) \leq c_{2} w_{2}(x)$, then $\mathbf{S}_{\text {sing }}\left(w_{1}, w_{2}\right)=\emptyset$, in particular, $\mathbf{S}_{\text {sing }}(w, w)=\emptyset$.

Examples 4.4. Let $w=w_{\alpha, \beta}$ given by (2.9), then

$$
\mathbf{S}_{\text {sing }}\left(w_{\alpha, \beta}\right)=\{0\}, \quad \alpha \neq 0,
$$

with $\mathbf{S}_{\text {sing }}\left(w_{\alpha, \beta}\right)=\emptyset$ if $\alpha=0$; thus

$$
\left|\mathbf{S}_{\text {sing }}\left(w_{\alpha, \beta}\right)\right|=0, \quad \alpha, \beta>-n .
$$

Assertion (4.1) can be seen as follows. Note that

$$
w_{\alpha, \beta}\left(Q_{\nu, m}\right) \sim 2^{-\nu n} \begin{cases}2^{-\nu \alpha} & \text { if } m=0, \\ \left|2^{-\nu} m\right|^{\alpha} & \text { if } 1 \leq|m|<2^{\nu}, \\ \left|2^{-\nu} m\right|^{\beta} & \text { if }|m| \geq 2^{\nu} .\end{cases}
$$

Hence we obtain for $x_{0}=0$ that

$$
\sup _{Q_{\nu, m} \ni x_{0}} \frac{w_{\alpha, \beta}\left(Q_{\nu, m}\right)}{\left|Q_{\nu, m}\right|} \geq \sup _{\nu \in \mathbf{N}_{0}} 2^{-\nu \alpha}=\infty \quad \text { for } \alpha<0, \text { that is, } 0 \in \mathbf{S}_{\infty}\left(w_{\alpha, \beta}\right),
$$

and

$$
\inf _{Q_{\nu, m} \ni x_{0}} \frac{w_{\alpha, \beta}\left(Q_{\nu, m}\right)}{\left|Q_{\nu, m}\right|} \leq \inf _{\nu \in \mathbf{N}_{0}} 2^{-\nu \alpha}=0 \quad \text { for } \alpha>0, \text { that is, } 0 \in \mathbf{S}_{0}\left(w_{\alpha, \beta}\right) .
$$

Conversely, straightforward reasoning gives for $\left|x_{0}\right| \geq 1$ that

$$
\frac{w_{\alpha, \beta}\left(Q_{\nu, m}\right)}{\left|Q_{\nu, m}\right|} \sim\left|x_{0}\right|^{\beta} \quad \text { for all } Q_{\nu, m} \ni x_{0} \text {, that is, } x_{0} \notin \mathbf{S}_{\text {sing }}\left(w_{\alpha, \beta}\right),
$$


and for $0<\left|x_{0}\right|<1$ that

$$
\sup _{Q_{\nu, m} \ni x_{0}} \frac{w_{\alpha, \beta}\left(Q_{\nu, m}\right)}{\left|Q_{\nu, m}\right|} \sim \max \left(1,\left|x_{0}\right|^{\alpha}\right)<\infty, \text { that is, } x_{0} \notin \mathbf{S}_{\infty}\left(w_{\alpha, \beta}\right),
$$

as well as

$$
\inf _{Q_{\nu, m} \ni x_{0}} \frac{w_{\alpha, \beta}\left(Q_{\nu, m}\right)}{\left|Q_{\nu, m}\right|} \sim \min \left(1,\left|x_{0}\right|^{\alpha}\right)>0, \text { that is, } x_{0} \notin \mathbf{S}_{0}\left(w_{\alpha, \beta}\right) .
$$

We return to our example $w_{\varkappa, \Gamma}$ introduced in (2.10) in Example 2.3 where $\Gamma$ is a $d$-set in $\mathbf{R}^{n}$ with $0<d<n$ and $\varkappa>-(n-d)$. Then one can prove similar to our above considerations, see also [16], that

$$
\mathbf{S}_{\text {sing }}\left(w_{\varkappa, \Gamma}\right)= \begin{cases}\Gamma=\mathbf{S}_{0}\left(w_{\varkappa, \Gamma}\right), \mathbf{S}_{\infty}\left(w_{\varkappa, \Gamma}\right)=\emptyset, & \text { if } \varkappa>0, \\ \emptyset=\mathbf{S}_{0}\left(w_{\varkappa, \Gamma}\right)=\mathbf{S}_{\infty}\left(w_{\varkappa, \Gamma}\right), & \text { if } \varkappa=0, \\ \Gamma=\mathbf{S}_{\infty}\left(w_{\varkappa, \Gamma}\right), \mathbf{S}_{0}\left(w_{\varkappa, \Gamma}\right)=\emptyset, & \text { if } \varkappa<0 .\end{cases}
$$

Note that $|\Gamma|=0$, cf. [39, Cor. 3.6], such that finally

$$
\left|\mathbf{S}_{\text {sing }}\left(w_{\varkappa, \Gamma}\right)\right|=0, \quad \varkappa>-(n-d) .
$$

Inspired by the above examples we assume in the sequel that $\mathbf{S}_{\text {sing }}\left(w_{1}, w_{2}\right)$ is bounded in $\mathbf{R}^{n}$. Moreover, another common feature of the above examples (4.2), (4.3), that is, $\left|\mathbf{S}_{\text {sing }}(w)\right|=0, w \in \mathscr{A}_{\infty}$, can be proved in general.

Proposition 4.5. For any $w_{1}, w_{2} \in \mathscr{A}_{\infty}$ the set $\mathbf{S}_{\text {sing }}\left(w_{1}, w_{2}\right)$ has Lebesgue measure 0, i.e., $\left|\mathbf{S}_{\text {sing }}\left(w_{1}, w_{2}\right)\right|=0$.

Proof. Step 1. It follows directly from the definition and elementary properties of inf and sup that

$$
\mathbf{S}_{0}\left(w_{1}, w_{2}\right) \subsetneq \mathbf{S}_{0}\left(w_{1}\right) \cup \mathbf{S}_{\infty}\left(w_{2}\right) \quad \text { and } \quad \mathbf{S}_{\infty}\left(w_{1}, w_{2}\right) \subsetneq \mathbf{S}_{\infty}\left(w_{1}\right) \cup \mathbf{S}_{0}\left(w_{2}\right) .
$$

So, it is sufficient to prove that for any $w \in \mathscr{A}_{\infty}$ the set $\mathbf{S}_{\text {sing }}(w)$ is of measure 0 , since $\mathbf{S}_{\text {sing }}\left(w_{1}, w_{2}\right)=\mathbf{S}_{0}\left(w_{1}, w_{2}\right) \cup \mathbf{S}_{\infty}\left(w_{1}, w_{2}\right)$. In the rest of the proof we will thus assume that $w_{1}=w \in \mathscr{A}_{\infty}$ and $w_{2} \equiv 1$.

Step 2. First we assume that $w \in \mathscr{A}_{1}$. We prove that $\mathbf{S}_{0}(w)=\emptyset$ and $\left|\mathbf{S}_{\infty}(w)\right|=0$. If $w \in \mathscr{A}_{1}$, then $w(x)$ is finite a.e. in $\mathbf{R}^{n}$ and by (2.3) there is a constant $C>0$ such that $M w(x) \leq C w(x)$ for a.e. $x \in \mathbf{R}^{n}$. Thus $\left|\mathbf{S}_{\infty}(w)\right|=0$. On the other hand, (2.3) implies for $x_{0} \in Q_{0, \ell}$ and some $\ell \in \mathbf{N}_{0}$ that

$$
\left|Q_{0, \ell}\right|^{-1} w\left(Q_{0, \ell}\right) \leq C \underset{Q_{0, \ell}}{\operatorname{essinf}} w(x) \leq C \inf _{Q_{0, \ell} \supset Q_{\nu, m} \ni x_{0}} \frac{w\left(Q_{\nu, m}\right)}{\left|Q_{\nu, m}\right|} .
$$

This gives $\mathbf{S}_{0}(w)=\emptyset$ and

$$
\left|\mathbf{S}_{\text {sing }}(w)\right|=0, \quad w \in \mathscr{A}_{1} .
$$

Step 3. Let $w(x)=v(x)^{1-p}$ for some $v \in \mathscr{A}_{1}$ and $1<p<\infty$, then $w \in \mathscr{A}_{p}$ in view of $(2.5)$ (with $w_{1} \equiv 1$ ). We show that $\mathbf{S}_{0}(v)=\mathbf{S}_{\infty}(w)$ and $\mathbf{S}_{\infty}(v)=\mathbf{S}_{0}(w)$. Note that this leads to $\mathbf{S}_{\text {sing }}(v)=\mathbf{S}_{\text {sing }}(w)$ immediately and thus by (4.5) to

$$
\left|\mathbf{S}_{\text {sing }}(w)\right|=0 \text { for } w=v^{1-p}, v \in \mathscr{A}_{1} .
$$

By (2.1) we obtain

$$
\frac{w(Q)}{|Q|} \leq C\left(\frac{v(Q)}{|Q|}\right)^{1-p}
$$


for any cube $Q \subset \mathbf{R}^{n}$. The weight $v$ belongs to the class $\mathscr{A}_{1}$ therefore (2.7) reads as

$$
\frac{v\left(Q_{0, \ell}\right)}{\left|Q_{0, \ell}\right|} \leq C \frac{v\left(Q_{\nu, m}\right)}{\left|Q_{\nu, m}\right|}
$$

for any cube $Q_{0, \ell}$ and $Q_{\nu, m}$ such that $Q_{\nu, m} \subset Q_{0, \ell}$. Thus the inequalities (4.7) and (4.8) imply that for any cube $Q_{0, \ell}$ there exists a positive constant $C>0$ such that

$$
\frac{w\left(Q_{\nu, m}\right)}{\left|Q_{\nu, m}\right|} \leq C
$$

for any $Q_{\nu, m} \subset Q_{0, \ell}$. This enables us to conclude $\mathbf{S}_{\infty}(w)=\emptyset=\mathbf{S}_{0}(v)$ in view of Step 2. It remains to prove $\mathbf{S}_{\infty}(v)=\mathbf{S}_{0}(w)$.

If $x \in \mathbf{S}_{\infty}(v)$, then by (4.7) and $p>1$ we get

$$
\inf _{Q_{\nu, m} \ni x} \frac{w\left(Q_{\nu, m}\right)}{\left|Q_{\nu, m}\right|} \leq C\left(\sup _{Q_{\nu, m} \ni x} \frac{v\left(Q_{\nu, m}\right)}{\left|Q_{\nu, m}\right|}\right)^{1-p}=0 .
$$

Thus $\mathbf{S}_{\infty}(v) \subset \mathbf{S}_{0}(w)$. Conversely, let $x \in \mathbf{S}_{0}(w)$, then for any $\varepsilon>0$ there is a cube $Q_{\nu, m}^{\varepsilon} \ni x$ such that $\frac{w\left(Q_{\nu, m}^{\varepsilon}\right)}{\left|Q_{\nu, m}^{\varepsilon}\right|}<\varepsilon$. Moreover, if $x \in Q_{0, \ell}$, then there exists a constant $c>0$ such that $\operatorname{ess}_{\sup } \operatorname{Qup}_{0, \ell} w(x) \leq c$ since $v \in \mathscr{A}_{1} \operatorname{implies}_{\operatorname{ess}} \inf _{Q_{0, \ell}} v(x)>C v\left(Q_{0, \ell}\right)$ by (4.4).

We apply Hölder's inequality with $p>1, \frac{1}{p}+\frac{1}{p^{\prime}}=1$, and get

$$
\begin{aligned}
1 & =\left|Q_{\nu, m}^{\varepsilon}\right|^{-1} \int_{Q_{\nu, m}^{\varepsilon}} w^{\frac{1}{p}}(x) v^{(p-1) \frac{1}{p}}(x) d x \leq\left(\frac{w\left(Q_{\nu, m}^{\varepsilon}\right)}{\left|Q_{\nu, m}^{\varepsilon}\right|}\right)^{1 / p}\left(\frac{v\left(Q_{\nu, m}^{\varepsilon}\right)}{\left|Q_{\nu, m}^{\varepsilon}\right|}\right)^{1 / p^{\prime}} \\
& \leq C \varepsilon^{1 / p}\left(\frac{v\left(Q_{\nu, m}^{\varepsilon}\right)}{\left|Q_{\nu, m}^{\varepsilon}\right|}\right)^{1 / p^{\prime}} .
\end{aligned}
$$

But this implies

$$
\sup _{Q_{\nu, m} \ni x} \frac{v\left(Q_{\nu, m}\right)}{\left|Q_{\nu, m}\right|}=\infty
$$

hence, $\mathbf{S}_{0}(w) \subset \mathbf{S}_{\infty}(v)$.

Step 4. Let $w \in \mathscr{A}_{\infty}$, then (2.4) and (2.5) imply the existence of some $1<p<\infty$ and $v_{1}, v_{2} \in \mathscr{A}_{1}$ with $w=v_{1}^{1-p} v_{2} \in \mathscr{A}_{p}$. It is sufficient to show that $\mathbf{S}_{\infty}(w) \subset \mathbf{S}_{\infty}\left(v_{2}\right)$ and $\mathbf{S}_{0}(w) \subset \mathbf{S}_{\infty}\left(v_{1}\right)$ since then (4.5), (4.6) imply

$$
\left|\mathbf{S}_{\text {sing }}(w)\right| \leq\left|\mathbf{S}_{0}(w)\right|+\left|\mathbf{S}_{\infty}(w)\right| \leq\left|\mathbf{S}_{\infty}\left(v_{1}\right)\right|+\left|\mathbf{S}_{\infty}\left(v_{2}\right)\right| \leq\left|\mathbf{S}_{\text {sing }}\left(v_{1}\right)\right|+\left|\mathbf{S}_{\text {sing }}\left(v_{2}\right)\right|=0 \text {. }
$$

Recall that by (4.4) for any cube $Q_{0, \ell}$ there are constants $c_{1}, c_{2}>0$ such that

$$
\underset{y \in Q_{0, \ell}}{\operatorname{essinf}} v_{2}(y)>c_{1} \quad \text { and } \quad \operatorname{essup}_{y \in Q_{0, \ell}} v_{1}^{1-p}(y)<c_{2} .
$$

Let $x \in \mathbf{S}_{\infty}(w)$ and $x \in Q_{\nu, m} \subset Q_{0, \ell}$. It follows by (4.9) that

$$
w\left(Q_{\nu, m}\right) \leq C v_{2}\left(Q_{\nu, m}\right)
$$

such that $x \in \mathbf{S}_{\infty}\left(v_{2}\right)$, in other words, $\mathbf{S}_{\infty}(w) \subset \mathbf{S}_{\infty}\left(v_{2}\right)$. Assume now $x \in \mathbf{S}_{0}(w)$ and $x \in Q_{\nu, m} \subset Q_{0, \ell}$, then the right-hand side of (4.9) implies

$$
w\left(Q_{\nu, m}\right) \geq C v_{1}^{1-p}\left(Q_{\nu, m}\right),
$$


that is, $x \in \mathbf{S}_{0}\left(v_{1}^{1-p}\right)=\mathbf{S}_{\infty}\left(v_{1}\right)$ in view of Step 3. This establishes $\mathbf{S}_{0}(w) \subset \mathbf{S}_{\infty}\left(v_{1}\right)$ and hence completes the proof.

Let $Q$ be some (closed) dyadic cube in $\mathbf{R}^{n}$ by which we mean a cube with sides parallel to the axes and of side length $L 2^{-\nu_{0}}$ for suitable $L \in \mathbf{N}_{0}$ and $\nu_{0} \in \mathbf{N}_{0}$ such that

$$
Q=\bigcup_{m \in I_{\nu_{0}}} Q_{\nu_{0}, m}=\bigcup_{\nu \geq \nu_{0}} \bigcup_{m \in I_{\nu}} Q_{\nu, m}
$$

for suitable finite index sets $I_{\nu} \subset \mathbf{Z}^{n}$. This ensures by the construction of the cubes $Q_{\nu, m}$ that we have at each level $\nu \geq \nu_{0}$ either $Q_{\nu, m} \subset Q$ or $Q_{\nu, m} \cap Q=\emptyset$. Let $B_{p, q}^{s}(Q, w)$ defined by restriction, that is, (2.16) with $\Omega=Q$, normed by (2.17). We first show that such a space 'ignores' the influence of a weight if the corresponding set of singularities $\mathbf{S}_{\text {sing }}\left(w_{1}, w_{2}\right)$ is at some distance from $Q$.

Proposition 4.6. Let $s \in \mathbf{R}, 0<p, q \leq \infty, w_{1}, w_{2} \in \mathscr{A}_{\infty}$, and assume $\operatorname{dist}\left(Q, \overline{\mathbf{S}_{\text {sing }}\left(w_{1}, w_{2}\right)}\right)>0$. Then

$$
B_{p, q}^{s}\left(Q, w_{1}\right)=B_{p, q}^{s}\left(Q, w_{2}\right),
$$

(in the sense of equivalent norms), in particular,

$$
B_{p, q}^{s}(Q, w)=B_{p, q}^{s}(Q) \quad \text { for } w \in \mathscr{A}_{\infty} \text { with } \operatorname{dist}\left(Q, \overline{\mathbf{S}_{\text {sing }}(w)}\right)>0 .
$$

Proof. Step 1. First we remark that one can define $B_{p, q}^{s}\left(Q, w_{i}\right)$ by the restriction of distributions belonging $B_{p, q}^{s}\left(\mathbf{R}^{n}, w_{i}\right)$ and supported in any open set $\Omega \subset \mathbf{R}^{n}$ such that $\bar{Q} \subset \Omega, i=1,2$. This follows easily from the fact that any smooth compactly supported function is a pointwise multiplier in $B_{p, q}^{s}\left(\mathbf{R}^{n}, w_{i}\right)$, cf. [31].

We choose an integer $\nu_{1}$ such that $\nu_{1} \geq \nu_{0}$ and $\operatorname{dist}\left(Q, \overline{\mathbf{S}_{\text {sing }}\left(w_{1}, w_{2}\right)}\right)>2 \sqrt{n} 2^{-\nu_{1}}$. We put

$$
I_{\nu_{1}}=\left\{m \in \mathbf{Z}^{n}: \overline{Q_{\nu_{1}, m}} \cap \bar{Q} \neq \emptyset\right\} \quad \text { and } \quad Q_{1}=\bigcup_{m \in I_{\nu_{1}}} Q_{\nu_{1}, m}
$$

and

$$
J_{\nu_{1}}=\left\{m \in \mathbf{Z}^{n}: \overline{Q_{\nu_{1}, m}} \cap \overline{Q_{1}} \neq \emptyset\right\} \quad \text { and } \quad Q_{2}=\bigcup_{m \in J_{\nu_{1}}} Q_{\nu_{1}, m}
$$

Then

$$
\bar{Q} \subset Q_{1} \subset \overline{Q_{1}} \subset Q_{2} \text { and } \operatorname{dist}\left(Q_{2}, \overline{\mathbf{S}_{\text {sing }}\left(w_{1}, w_{2}\right)}\right)>0 .
$$

Let $\widetilde{B}_{p, q}^{s}\left(Q_{1}, w_{i}\right)=\left\{f \in B_{p, q}^{s}\left(Q_{1}, w_{i}\right): \operatorname{supp} f \subset Q_{1}\right\}$. Then $\widetilde{B}_{p, q}^{s}\left(Q_{1}, w_{i}\right) \hookrightarrow$ $\widetilde{B}_{p, q}^{s}\left(Q_{1}, w_{j}\right)$ implies $B_{p, q}^{s}\left(Q, w_{i}\right) \stackrel{\hookrightarrow}{\hookrightarrow} B_{p, q}^{s}\left(Q, w_{j}\right), i, j=1,2$.

Now we apply Theorem 2.6 and Proposition 3.1 with $s_{1}=s_{2}=s, p_{1}=p_{2}=p$, $q_{1}=q_{2}=q$, hence $p^{*}=q^{*}=\infty$. Since the wavelets used in Theorem 2.6 are compactly supported we obtain that

$$
\widetilde{B}_{p, q}^{s}\left(Q_{1}, w_{1}\right) \hookrightarrow \widetilde{B}_{p, q}^{s}\left(Q_{1}, w_{2}\right) \quad \text { if } \sup _{Q_{\nu, m} \subset Q_{2}} \frac{w_{2}\left(Q_{\nu, m}\right)}{w_{1}\left(Q_{\nu, m}\right)}<\infty
$$

that is,

$$
\widetilde{B}_{p, q}^{s}\left(Q_{1}, w_{1}\right) \hookrightarrow \widetilde{B}_{p, q}^{s}\left(Q_{1}, w_{2}\right) \text { if } \inf _{Q_{\nu, m} \subset Q_{2}} \frac{w_{1}\left(Q_{\nu, m}\right)}{w_{2}\left(Q_{\nu, m}\right)}>0
$$


Similarly, interchanging the roles of $w_{1}$ and $w_{2}$, we get

$$
\widetilde{B}_{p, q}^{s}\left(Q_{1}, w_{2}\right) \hookrightarrow \widetilde{B}_{p, q}^{s}\left(Q_{1}, w_{1}\right) \quad \text { if } \sup _{Q_{\nu, m} \subset Q_{2}} \frac{w_{1}\left(Q_{\nu, m}\right)}{w_{2}\left(Q_{\nu, m}\right)}<\infty .
$$

Step 2. It remains to show that $\operatorname{dist}\left(Q_{2}, \overline{\mathbf{S}_{\text {sing }}\left(w_{1}, w_{2}\right)}\right)>0$ implies

$$
0<\inf _{Q_{\nu, m} \subset Q_{2}} \frac{w_{1}\left(Q_{\nu, m}\right)}{w_{2}\left(Q_{\nu, m}\right)} \leq \sup _{Q_{\nu, m} \subset Q_{2}} \frac{w_{1}\left(Q_{\nu, m}\right)}{w_{2}\left(Q_{\nu, m}\right)}<\infty .
$$

We proceed by contradiction and first assume that

$$
\inf _{Q_{\nu, m} \subset Q_{2}} \frac{w_{1}\left(Q_{\nu, m}\right)}{w_{2}\left(Q_{\nu, m}\right)}=0 .
$$

Then by our assumption on $Q_{2}$ there is at least some $m_{0} \in J_{\nu_{1}}$ such that

$$
\inf _{Q_{\nu, m} \subset Q_{\nu_{1}, m_{0}}} \frac{w_{1}\left(Q_{\nu, m}\right)}{w_{2}\left(Q_{\nu, m}\right)}=0 .
$$

There are $2^{n}$ sub-cubes $Q_{\nu_{1}+1, m} \subset Q_{\nu_{1}, m_{0}}$ of side length $2^{-\left(\nu_{1}+1\right)}$ where again there must exist at least one number $m_{1} \in J_{\nu_{1}+1}$ such that

$$
\inf _{Q_{\nu, m} \subset Q_{\nu_{1}+1, m_{1}}} \frac{w_{1}\left(Q_{\nu, m}\right)}{w_{2}\left(Q_{\nu, m}\right)}=0 .
$$

By this construction we obtain a decreasing sequence of cubes $\left\{Q_{\nu_{1}+j, m_{j}}\right\}_{j \in \mathbf{N}_{0}}$ with $Q_{2} \supset Q_{\nu_{1}, m_{0}} \supset Q_{\nu_{1}+1, m_{1}} \supset \cdots \supset Q_{\nu_{1}+j, m_{j}} \supset \cdots$, and

$$
\inf _{Q_{\nu, m} \subset Q_{\nu_{1}+j, m_{j}}} \frac{w_{1}\left(Q_{\nu, m}\right)}{w_{2}\left(Q_{\nu, m}\right)}=0 .
$$

Since $Q_{2}$ is compact, there exists some $x_{1} \in \bigcap_{j \in \mathbf{N}_{0}} Q_{\nu_{1}+j, m_{j}} \subset Q_{2}$ with

$$
\inf _{Q_{\nu, m} \ni x_{1}} \frac{w_{1}\left(Q_{\nu, m}\right)}{w_{2}\left(Q_{\nu, m}\right)}=0 \quad \text { such that } x_{1} \in \mathbf{S}_{0}\left(w_{1}, w_{2}\right) \cap Q_{2} .
$$

Consequently, $\mathbf{S}_{\text {sing }}\left(w_{1}, w_{2}\right) \cap Q_{2} \neq \emptyset$ which contradicts $\operatorname{dist}\left(Q_{2}, \overline{\mathbf{S}_{\text {sing }}\left(w_{1}, w_{2}\right)}\right)>0$. The argument in case of $\sup _{Q_{\nu, m} \subset Q_{2}} \frac{w_{1}\left(Q_{\nu, m}\right)}{w_{2}\left(Q_{\nu, m}\right)}=\infty$ is parallel and leads to the existence of some $x^{1} \in \mathbf{S}_{\infty}\left(w_{1}, w_{2}\right) \cap Q_{2}$, which again contradicts $\operatorname{dist}\left(Q_{2}, \overline{\mathbf{S}_{\text {sing }}\left(w_{1}, w_{2}\right)}\right)>0$.

4.2. Entropy and approximation numbers for one-sided weighted situations. We first deal with the situation $w_{1} \equiv w$ and $w_{2} \equiv 1$ which corresponds to (3.6). Recall Example 4.1 for the special weight of type (2.9).

Theorem 4.7. Let $w \in \mathscr{A}_{\infty}$ with $r_{w}$ given by (2.8) and $\mathbf{S}_{\text {sing }}(w)$ bounded. Let the parameters be given by (3.1), (3.5), (3.7), and assume

$$
\delta>\frac{n}{p^{*}}+\frac{n}{p_{1}}\left(r_{w}-1\right) .
$$

Let $Q$ be some sufficiently large cube with $\overline{\mathbf{S}_{\text {sing }}(w)} \subsetneq \stackrel{Q}{q}$. Then

$$
\mathrm{id}_{w}^{Q}: A_{p_{1}, q_{1}}^{s_{1}}(Q, w) \hookrightarrow A_{p_{2}, q_{2}}^{s_{2}}(Q)
$$

is compact with

$$
e_{k}\left(\operatorname{id}_{w}^{Q}\right) \sim k^{-\frac{s_{1}-s_{2}}{n}}, \quad k \in \mathbf{N}
$$


Proof. Step 1. First note that it is sufficient to deal with the case $A=B$ in (4.12), i.e., with Besov spaces, in view of (3.26). Our assumptions on $\mathbf{S}_{\text {sing }}(w)$ and $Q$ imply the existence of some smaller cube $\widetilde{Q} \subset Q$ with $\operatorname{dist}\left(\widetilde{Q}, \overline{\mathbf{S}_{\text {sing }}(w)}\right)>0$. This leads by Proposition 4.6 to

$$
0<c_{1} \leq w(\widetilde{Q}) \leq c_{2}|\widetilde{Q}| \leq c_{3}<\infty \quad \text { and } \quad B_{p_{1}, q_{1}}^{s_{1}}(\widetilde{Q}, w)=B_{p_{1}, q_{1}}^{s_{1}}(\widetilde{Q})
$$

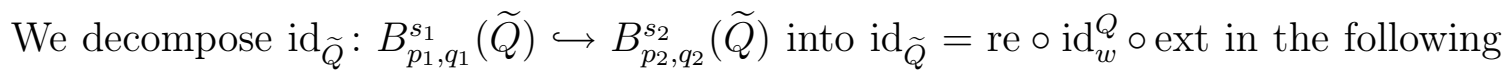
way,

$$
\begin{array}{ccc}
B_{p_{1}, q_{1}}^{s_{1}}(\widetilde{Q})=B_{p_{1}, q_{1}}^{s_{1}}(\widetilde{Q}, w) & \stackrel{\text { ext }}{\longrightarrow} & B_{p_{1}, q_{1}}^{s_{1}}(Q, w) \\
\operatorname{id}_{\widetilde{Q}} \downarrow & \downarrow \mathrm{id}_{w}^{Q} \\
B_{p_{2}, q_{2}}^{s_{2}}(\widetilde{Q}) & \longleftarrow \text { re } & B_{p_{2}, q_{2}}^{s_{2}}(Q)
\end{array}
$$

where re and ext are the usual restriction and extension operators. Since they are bounded, the multiplicativity of entropy numbers leads to

$$
e_{k}\left(\operatorname{id}_{\widetilde{Q}}\right) \leq c e_{k}\left(\mathrm{id}_{w}^{Q}\right), \quad k \in \mathbf{N}
$$

Note that $\delta>\frac{n}{p^{*}}$ as a consequence of (4.11). Therefore, we can apply the unweighted result for entropy numbers in [10, Thm. 3.3.3/2] which yields

$$
e_{k}\left(\operatorname{id}_{\widetilde{Q}}\right) \geq c^{\prime} k^{-\frac{s_{1}-s_{2}}{n}}, \quad k \in \mathbf{N}
$$

leading to the lower estimate in (4.13).

Step 2. We deal with the estimate from above and begin with the case $w \in \mathscr{A}_{1}$ such that (4.11) reads as $\delta>\frac{n}{p^{*}}$. Similar to the proof of Proposition 4.6 we know that

$$
\operatorname{id}_{1}: B_{p_{1}, q_{1}}^{s_{1}}(Q, w) \hookrightarrow B_{p_{1}, q_{1}}^{s_{1}}(Q) \text { if and only if } \inf _{Q_{\nu, m} \subset Q} \frac{w\left(Q_{\nu, m}\right)}{\left|Q_{\nu, m}\right|}>0 .
$$

Note that

$$
\inf _{Q_{0, l} \subset Q} \frac{w\left(Q_{0, l}\right)}{\left|Q_{0, l}\right|}=\inf _{Q_{0, l} \subset Q} w\left(Q_{0, l}\right) \geq c>0
$$

(as there are only finitely many such cubes $Q_{0, l} \subset Q$ ), we now apply (2.7) with $p=1$ and $Q_{\nu, m} \subset Q_{0, l}$ for some $l \in \mathbf{Z}^{n}$, thus

$$
\inf _{Q_{\nu, m} \subset Q} \frac{w\left(Q_{\nu, m}\right)}{\left|Q_{\nu, m}\right|} \geq c^{\prime} \inf _{Q_{0, l} \subset Q} \frac{w\left(Q_{0, l}\right)}{\left|Q_{0, l}\right|} \geq c^{\prime \prime},
$$

such that $\mathbf{S}_{0}(w)=\emptyset$ and $\mathbf{S}_{\text {sing }}(w)=\mathbf{S}_{\infty}(w)$ with $w(x) \geq C>0$ a.e. in $Q$. This implies the continuity of $\mathrm{id}_{1}$ and the counterpart of (4.14) looks like

$$
\begin{array}{r}
B_{p_{1}, q_{1}}^{s_{1}}(Q, w) \stackrel{\mathrm{id}_{1}}{\longrightarrow} B_{p_{1}, q_{1}}^{s_{1}}(Q) \\
\operatorname{id}_{w}^{Q} \searrow \underset{B_{p_{2}, q_{2}}^{s_{2}}(Q)}{\swarrow \mathrm{id}_{Q}}
\end{array} \quad \text { that is, } \mathrm{id}_{w}^{Q}=\mathrm{id}_{Q} \circ \mathrm{id}_{1}
$$

such that the multiplicativity of entropy numbers gives

$$
e_{k}\left(\operatorname{id}_{w}^{Q}\right) \leq c e_{k}\left(\operatorname{id}_{Q}\right) \leq c^{\prime} k^{-\frac{s_{1}-s_{2}}{n}}, \quad k \in \mathbf{N},
$$

where we used $\delta>\frac{n}{p^{*}}$ and the unweighted result [10, Thm. 3.3.3/2] for $\operatorname{id}_{Q}$. 
Step 3. Assume now $w \in \mathscr{A}_{p}, p>1$. By (4.11) we may choose some $r>r_{w} \geq 1$ such that

$$
\delta>\frac{n}{p^{*}}+\frac{n}{p_{1}}(r-1)>\frac{n}{p^{*}}+\frac{n}{p_{1}}\left(r_{w}-1\right)
$$

and $w \in \mathscr{A}_{r}$. This implies

$$
\left(\int_{Q} w(x) d x\right)^{1 / r}\left(\int_{Q} w(x)^{-r^{\prime} / r} d x\right)^{1 / r^{\prime}} \leq A|Q|<\infty
$$

and consequently,

$$
\left(\int_{Q} w(x)^{-r^{\prime} / r} d x\right)^{1 / r^{\prime}} \leq C<\infty
$$

Thus Hölder's inequality ensures $L_{p_{1}}(Q, w) \hookrightarrow L \frac{p_{1}}{r}(Q)$ since

$$
\begin{aligned}
\left\|f \mid L_{\frac{p_{1}}{r}}(Q)\right\| & =\left\|f w^{\frac{1}{p_{1}}} w^{-\frac{1}{p_{1}}} \mid L_{\frac{p_{1}}{r}}(Q)\right\| \\
& \leq\left\|f\left|L_{p_{1}}(Q, w)\|\| w^{-\frac{1}{r}}\right| L_{r^{\prime}}(Q)\right\|^{\frac{r}{p_{1}}} \leq C\left\|f \mid L_{p_{1}}(Q, w)\right\| .
\end{aligned}
$$

In a similar way as in Step 2 we get

$$
\operatorname{id}_{1}^{r}: B_{p_{1}, q_{1}}^{s_{1}}(Q, w) \hookrightarrow B_{\frac{p_{1}}{r}, q_{1}}^{s_{1}}(Q),
$$

and (4.15) is replaced by

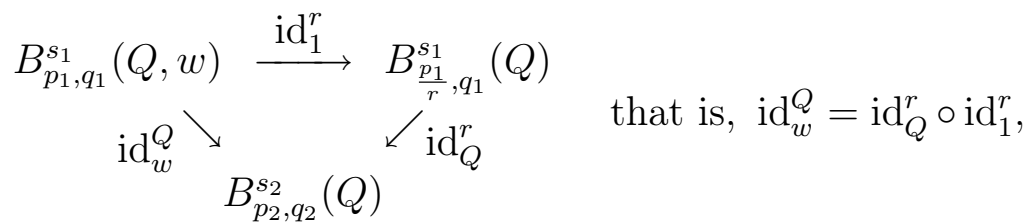

leading to $e_{k}\left(\operatorname{id}_{w}^{Q}\right) \leq c e_{k}\left(\operatorname{id}_{Q}^{r}\right), k \in \mathbf{N}$. The (unweighted) embedding $\operatorname{id}_{Q}^{r}$ is compact with $e_{k}\left(\mathrm{id}_{Q}^{r}\right) \sim k^{-\frac{s_{1}-s_{2}}{n}}$ if

$$
s_{1}-s_{2}-n\left(\frac{r}{p_{1}}-\frac{1}{p_{2}}\right)_{+}>0 .
$$

If $p_{1} \leq p_{2}$, then $p^{*}=\infty$ and (4.16) gives the desired estimate, since

$$
s_{1}-s_{2}-n\left(\frac{r}{p_{1}}-\frac{1}{p_{2}}\right)_{+}=s_{1}-s_{2}-\frac{n r}{p_{1}}+\frac{n}{p_{2}}=\delta-\frac{n}{p_{1}}(r-1)>0 .
$$

When $p_{1}>p_{2}$, then either $1 \leq r_{w}<r \leq \frac{p_{1}}{p_{2}}$ such that

$$
s_{1}-s_{2}-n\left(\frac{r}{p_{1}}-\frac{1}{p_{2}}\right)_{+}=s_{1}-s_{2}>\frac{n}{p_{1}}(r-1)>0,
$$

or $1<\frac{p_{1}}{p_{2}} \leq r_{w}<r$ such that

$$
s_{1}-s_{2}-n\left(\frac{r}{p_{1}}-\frac{1}{p_{2}}\right)_{+}=\delta-\frac{n}{p_{1}}(r-1)>0 .
$$

This concludes the proof. 
Remark 4.8. In the unweighted case $w \equiv 1$ we have the asymptotic behaviour of entropy numbers (4.13) whenever $\delta>\frac{n}{p^{*}}$, see [10, Thm. 3.3.3/2]. This fits well together with the above condition (4.11), since we have $r_{w}=1$ in the unweighted case, see Example 2.3.

The above result has some immediate consequences for the asymptotic estimate of the corresponding approximation numbers. Recall that in case of bounded domains the parallel result to [10, Thm. 3.3.3/2] was achieved in [10, Thm. 3.3.4] with the extension in [7]. In case of $\delta>\frac{n}{p^{*}}$ it reads as

$$
a_{k}\left(A_{p_{1}, q_{1}}^{s_{1}}(\Omega) \hookrightarrow A_{p_{2}, q_{2}}^{s_{2}}(\Omega)\right) \sim k^{-\varkappa}, \quad k \in \mathbf{N},
$$

where

$$
\varkappa=\frac{\delta}{n}-\frac{1}{p^{*}}+\left(\frac{\min \left(p_{1}^{\prime}, p_{2}\right)}{2}-1\right)_{+} \cdot \min \left(\frac{\delta}{n}, \frac{1}{\min \left(p_{1}^{\prime}, p_{2}\right)}\right),
$$

with the additional assumption that $\left(p_{1}, p_{2}\right) \neq(1, \infty)$ and $\delta \neq \frac{n}{\min \left(p_{1}^{\prime}, p_{2}\right)}$ in case of $p_{1}<2<p_{2}$. Note that this coincides with Example 4.1(iii) when $\beta \rightarrow \infty$.

Corollary 4.9. Let $w \in \mathscr{A}_{\infty}$ with $r_{w}$ given by $(2.8)$ and $\mathbf{S}_{\text {sing }}(w)$ bounded. Let the parameters be given by (3.7) with (4.11). Let $Q$ be some sufficiently large cube with $\overline{\mathbf{S}_{\text {sing }}(w)} \subsetneq \stackrel{Q}{Q}$ and $\operatorname{id}_{w}^{Q}$ the compact embedding

$$
\operatorname{id}_{w}^{Q}: A_{p_{1}, q_{1}}^{s_{1}}(Q, w) \hookrightarrow A_{p_{2}, q_{2}}^{s_{2}}(Q) .
$$

(i) If $w \in \mathscr{A}_{1}$, then

$$
a_{k}\left(\operatorname{id}_{w}^{Q}\right) \sim k^{-\varkappa}, \quad k \in \mathbf{N}
$$

with $\varkappa$ given by $(4.19)$.

(ii) If $w \in \mathscr{A}_{p}$ with $p>1$, then there exists some $c>0$ such that for all $k \in \mathbf{N}$,

$$
a_{k}\left(\operatorname{id}_{w}^{Q}\right) \geq c k^{-\varkappa}
$$

where $\varkappa$ is given by (4.19). In particular, for $p_{1}>r_{w} p_{2} \geq p_{2}$, then

$$
a_{k}\left(\operatorname{id}_{w}^{Q}\right) \sim k^{-\frac{s_{1}-s_{2}}{n}}, \quad k \in \mathbf{N} .
$$

Proof. Again we may restrict ourselves to the case $A=B$ in view of (3.26). Inspecting the above proof we find that Steps 1 and 2 provide for $w \in \mathscr{A}_{1}$ that

$$
a_{k}\left(\operatorname{id}_{w}^{Q}\right) \sim a_{k}\left(\operatorname{id}_{\Omega}: B_{p_{1}, q_{1}}^{s_{1}}(\Omega) \hookrightarrow B_{p_{2}, q_{2}}^{s_{2}}(\Omega)\right), \quad k \in \mathbf{N},
$$

where $\Omega=\widetilde{Q}$ or $\Omega=Q$, neglecting the dependence of the constants upon the domain (as usual). This proves (i).

In case of $w \in \mathscr{A}_{p}, p>1$, we get the two-sided estimate (in the above interpretation)

$$
c_{1} a_{k}\left(\operatorname{id}_{\Omega}: B_{p_{1}, q_{1}}^{s_{1}}(\Omega) \hookrightarrow B_{p_{2}, q_{2}}^{s_{2}}(\Omega)\right) \leq a_{k}\left(\mathrm{id}_{w}^{Q}\right) \leq c_{2} a_{k}\left(\operatorname{id}_{\Omega}^{r}: B_{\frac{p_{1}}{r}, q_{1}}^{s_{1}}(\Omega) \hookrightarrow B_{p_{2}, q_{2}}^{s_{2}}(\Omega)\right)
$$

where $r$ is appropriately chosen according to (4.16). Plainly this gives (4.20) in view of (4.18). Moreover, when $p_{1}>r_{w} p_{2} \geq p_{2}$, then $\varkappa=\frac{s_{1}-s_{2}}{n}$ in (4.19) and (4.18) reads as

$$
a_{k}\left(B_{p_{1}, q_{1}}^{s_{1}}(\Omega) \hookrightarrow B_{p_{2}, q_{2}}^{s_{2}}(\Omega)\right) \sim k^{-\frac{s_{1}-s_{2}}{n}}, \quad k \in \mathbf{N}, p_{2}<p_{1}, s_{1}>s_{2} .
$$


This gives the lower estimate in (4.21) with $\Omega=\widetilde{Q}$. Conversely, we choose again $r$ appropriate, i.e., $1 \leq r_{w}<r<\frac{p_{1}}{p_{2}}$, such that (4.18) with $\Omega=Q$ completes the upper estimate in (4.21).

Remark 4.10. If $w \in \mathscr{A}_{p}$ with $p>1$, and $p_{1} \leq r_{w} p_{2}$, then one can only prove two-sided estimates by the above method. For instance, let $0<p_{1} \leq p_{2} \leq 2$ or $2 \leq 2 r_{w}<p_{1} \leq p_{2}$, then there is some positive $c>0$ and for any $\varepsilon>0$ some number $c_{\varepsilon}>0$ such that

$$
c k^{-\frac{\delta}{n}} \leq a_{k}\left(\mathrm{id}_{w}^{Q}\right) \leq c_{\varepsilon} k^{-\frac{\delta}{n}+\frac{r_{w}-1}{p_{1}}+\varepsilon}, \quad k \in \mathbf{N}
$$

and for $p_{2} \leq p_{1} \leq r_{w} p_{2}$ with either $p_{1}>2 r_{w} \geq 2$ or $p_{2} \leq 2$, then

$$
c k^{-\frac{s_{1}-s_{2}}{n}} \leq a_{k}\left(\mathrm{id}_{w}^{Q}\right) \leq c_{\varepsilon} k^{-\frac{s_{1}-s_{2}}{n}+\left(\frac{r_{w}}{p_{1}}-\frac{1}{p_{2}}\right)+\varepsilon}, \quad k \in \mathbf{N} .
$$

Similarly it works for all other cases, but we do not explicate it since the outcome is not yet satisfying.

\subsection{Entropy and approximation numbers for double-sided weighted} situations. We study the corresponding double-sided weighted situation now, recall Corollaries 3.12, 3.14, 3.15. The counterpart of Example 4.1 is the following, cf. [17, Thm. 3.6, Rem. 4.4].

Example 4.11. Let the parameters satisfy (3.9) and let the weight $w \in \mathscr{A}_{\infty}$ be of type (2.9) with

$$
\beta>0, p_{1}<p_{2}, \quad \delta>\max (\alpha, 0)\left(\frac{1}{p_{1}}-\frac{1}{p_{2}}\right) .
$$

(i) If $\beta\left(\frac{1}{p_{1}}-\frac{1}{p_{2}}\right) \neq \delta$, then for all $k \in \mathbf{N}$,

$$
e_{k}\left(A_{p_{1}, q_{1}}^{s_{1}}\left(\mathbf{R}^{n}, w\right) \hookrightarrow A_{p_{2}, q_{2}}^{s_{2}}\left(\mathbf{R}^{n}, w\right)\right) \sim k^{-\min \left(\frac{s_{1}-s_{2}}{n},\left(1+\frac{\beta}{n}\right)\left(\frac{1}{p_{1}}-\frac{1}{p_{2}}\right)\right) .}
$$

(ii) If $\beta\left(\frac{1}{p_{1}}-\frac{1}{p_{2}}\right)=\delta$ and $\tau=\frac{s_{1}-s_{2}}{n}+\frac{1}{q_{2}}-\frac{1}{q_{1}} \neq 0$, then for all $k \in \mathbf{N}$,

$$
e_{k}\left(B_{p_{1}, q_{1}}^{s_{1}}\left(\mathbf{R}^{n}, w\right) \hookrightarrow B_{p_{2}, q_{2}}^{s_{2}}\left(\mathbf{R}^{n}, w\right)\right) \sim k^{-\frac{s_{1}-s_{2}}{n}}(1+\log k)^{\max (\tau, 0)} .
$$

(iii) Let $\beta\left(\frac{1}{p_{1}}-\frac{1}{p_{2}}\right) \neq \delta$, then for all $k \in \mathbf{N}$,

$$
a_{k}\left(A_{p_{1}, q_{1}}^{s_{1}}\left(\mathbf{R}^{n}, w\right) \hookrightarrow A_{p_{2}, q_{2}}^{s_{2}}\left(\mathbf{R}^{n}, w\right)\right) \sim k^{-\varkappa},
$$

where

$$
\varkappa= \begin{cases}\min \left(\frac{\delta}{n}, \frac{\beta}{n}\left(\frac{1}{p_{1}}-\frac{1}{p_{2}}\right)\right) & \text { if } 0<p_{1} \leq p_{2} \leq 2, \text { or } 2 \leq p_{1} \leq p_{2} \leq \infty, \\ \min \left(\frac{\delta}{n}, \frac{\beta}{n}\left(\frac{1}{p_{1}}-\frac{1}{p_{2}}\right)\right)+\frac{1}{2}-\frac{1}{\min \left(p_{1}^{\prime}, p_{2}\right)} & \text { if } 0<p_{1}<2<p_{2} \leq \infty, \text { with }\left(p_{1}, p_{2}\right) \neq \\ & (1, \infty) \text { and } \min \left(\beta\left(\frac{1}{p_{1}}-\frac{1}{p_{2}}\right), \delta\right)>\frac{n}{\min \left(p_{1}^{\prime}, p_{2}\right)}, \\ \min \left(\frac{\delta}{n}, \frac{\beta}{n}\left(\frac{1}{p_{1}}-\frac{1}{p_{2}}\right)\right) \cdot \frac{\min \left(p_{1}^{\prime}, p_{2}\right)}{2} & \text { if } 0<p_{1}<2<p_{2} \leq \infty, \text { with }\left(p_{1}, p_{2}\right) \neq \\ & (1, \infty) \text { and } \min \left(\beta\left(\frac{1}{p_{1}}-\frac{1}{p_{2}}\right), \delta\right)<\frac{n}{\min \left(p_{1}^{\prime}, p_{2}\right)} .\end{cases}
$$

Recall $\mathbf{S}_{\text {sing }}(w, w)=\emptyset, \mathbf{S}_{\text {sing }}(w)=\mathbf{S}_{\text {sing }}(w, 1)$. The counterpart of Theorem 4.7 and Corollary 4.9 reads as follows. 
Theorem 4.12. Let $w \in \mathscr{A}_{\infty}$ with $r_{w}$ given by $(2.8)$ and $\mathbf{S}_{\text {sing }}(w)$ bounded. Let the parameters be given by (3.9) with $p_{1} \leq p_{2}$, and assume

$$
\delta>\left(r_{w}-1\right)\left(\frac{n}{p_{1}}-\frac{n}{p_{2}}\right) .
$$

Let $Q$ be some sufficiently large cube with $\overline{\mathbf{S}_{\text {sing }}(w)} \subsetneq \stackrel{Q}{\text {. Then }}$

$$
\operatorname{id}_{w w}^{Q}: A_{p_{1}, q_{1}}^{s_{1}}(Q, w) \hookrightarrow A_{p_{2}, q_{2}}^{s_{2}}(Q, w)
$$

is compact with

$$
e_{k}\left(\operatorname{id}_{w w}^{Q}\right) \sim k^{-\frac{s_{1}-s_{2}}{n}}, \quad k \in \mathbf{N}
$$

and

$$
a_{k}\left(\operatorname{id}_{w w}^{Q}\right) \sim k^{-\varkappa}, \quad k \in \mathbf{N},
$$

if $\delta \neq \frac{n}{\min \left(p_{1}^{\prime}, p_{2}\right)}$, where $\varkappa$ is defined in (4.19).

Proof. Step 1. First note that it is sufficient to deal with the case $A=B$ in (4.12), i.e., with Besov spaces, in view of (3.26). We proceed similar to the proof of

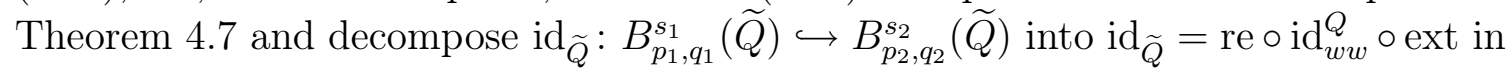
the following way,

$$
\begin{array}{cccc}
B_{p_{1}, q_{1}}^{s_{1}}(\widetilde{Q})=B_{p_{1}, q_{1}}^{s_{1}}(\widetilde{Q}, w) & \stackrel{\text { ext }}{\longrightarrow} & B_{p_{1}, q_{1}}^{s_{1}}(Q, w) \\
\operatorname{id}_{\widetilde{Q}} \downarrow & & \downarrow \mathrm{id}_{w w}^{Q} \\
B_{p_{2}, q_{2}}^{s_{2}}(\widetilde{Q})=B_{p_{2}, q_{2}}^{s_{2}}(\widetilde{Q}, w) & \longleftarrow \text { re } & B_{p_{2}, q_{2}}^{s_{2}}(Q, w)
\end{array}
$$

where we applied Proposition 4.6 with $w_{1} \equiv 1, w_{2} \equiv w$ in the left-hand part of the diagram. This leads to

$$
e_{k}\left(\operatorname{id}_{\widetilde{Q}}\right) \leq c e_{k}\left(\operatorname{id}_{w w}^{Q}\right), \quad a_{k}\left(\operatorname{id}_{\widetilde{Q}}\right) \leq c a_{k}\left(\operatorname{id}_{w w}^{Q}\right), \quad k \in \mathbf{N} .
$$

Since $\delta>0$ by (4.22), we can apply the unweighted result for entropy numbers in [10, Thm. 3.3.3/2] which yields

$$
e_{k}\left(\operatorname{id}_{\widetilde{Q}}\right) \geq c^{\prime} k^{-\frac{s_{1}-s_{2}}{n}}, \quad k \in \mathbf{N}
$$

leading to the lower estimate in (4.24). As for the counterpart in (4.25) we use (4.18) with (4.19).

Step 2. We prove the estimates from above. We need the following closed subspaces of the sequence spaces $b_{p, q}^{\sigma}(w)$ from (2.19),

$$
\widetilde{b}_{p, q}^{\sigma}(w):=\left\{\lambda=\left\{\lambda_{\nu, m}\right\}_{\nu, m}: \lambda_{\nu, m} \in b_{p, q}^{\sigma}(w), \lambda_{\nu, m}=0 \text { if }|m|>c 2^{\nu}\right\} .
$$


Here $c$ is a positive constant depending on the context. First we prove that there exist bounded linear operators $\widetilde{\mathrm{T}}$ and $\widetilde{\mathrm{S}}$ such that the following diagram is commutative,

$$
\begin{aligned}
& B_{p_{1}, q_{1}}^{s_{1}}(Q, w) \stackrel{\widetilde{\mathrm{T}}}{\longrightarrow} \widetilde{b}_{p_{1}, q_{1}}^{\sigma_{1}}(w) \\
& \operatorname{id}_{w w}^{Q} \downarrow \quad \downarrow \operatorname{Id}_{w w}^{Q} \\
& B_{p_{2}, q_{2}}^{s_{2}}(Q, w) \longleftarrow \widetilde{\mathrm{S}} \widetilde{b}_{p_{2}, q_{2}}^{\sigma_{2}}(w) .
\end{aligned}
$$

Let $r Q$ denote the cube concentric with $Q$ and of size $r$ times the size of $Q, r>0$. Since $\overline{\mathbf{S}_{\text {sing }}(w)} \subsetneq \stackrel{Q}{ }$ there exists some $r_{0}<1$ such that $\overline{\mathbf{S}_{\text {sing }}(w)} \subsetneq r_{0} Q$. We choose a number $r_{1}$ such that $r_{0}<r_{1}<1$. Assume that $\varphi, \psi \in C^{\infty}\left(\mathbf{R}^{n}\right)$ are compactly supported smooth functions such that

$$
\begin{aligned}
& \operatorname{supp} \varphi \subset r_{1} Q, \varphi(x)=1 \text { if } x \in r_{0} Q, \\
& \operatorname{supp} \psi \subset 2 Q, \psi(x)=1 \text { if } x \in \frac{3}{2} Q .
\end{aligned}
$$

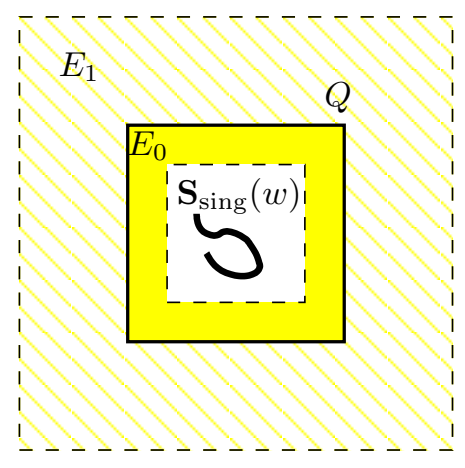

If $f \in B_{p_{1}, q_{1}}^{s_{1}}(Q, w)$, then also $f-\varphi f \in B_{p_{1}, q_{1}}^{s_{1}}(Q, w)$ and

$$
\left\|f-\varphi f\left|B_{p_{1}, q_{1}}^{s_{1}}(Q, w)\|\leq C\| f\right| B_{p_{1}, q_{1}}^{s_{1}}(Q, w)\right\|,
$$

since $\varphi$ is a pointwise multiplier in the space $B_{p_{1}, q_{1}}^{s_{1}}\left(\mathbf{R}^{n}, w\right)$, cf. [31]. But $\operatorname{supp}(f-$ $\varphi f) \subset E_{0}=Q \backslash r_{0} Q$ and $E_{0}$ is a Lipschitz domain with $\operatorname{dist}\left(E_{0}, \overline{\mathbf{S}_{\text {sing }}(w)}\right)>0$, so one can prove in the same way as in Proposition 4.6 that

$$
f-\varphi f \in B_{p_{1}, q_{1}}^{s_{1}}\left(E_{0}, w\right)=B_{p_{1}, q_{1}}^{s_{1}}\left(E_{0}\right)
$$

(in the sense of equivalent norms). Extending $f-\varphi f$ by zero onto $r_{0} Q$ we get $f-\varphi f \in B_{p_{1}, q_{1}}^{s_{1}}(Q)$ and by $(4.28)$,

$$
\left\|f-\varphi f\left|B_{p_{1}, q_{1}}^{s_{1}}(Q)\|\leq C\| f\right| B_{p_{1}, q_{1}}^{s_{1}}(Q, w)\right\| .
$$

Let $\operatorname{ext}_{Q}: B_{p_{1}, q_{1}}^{s_{1}}(Q) \rightarrow B_{p_{1}, q_{1}}^{s_{1}}\left(\mathbf{R}^{n}\right)$ denote a linear extension operator. The distribution $\psi \operatorname{ext}_{Q}(f-\varphi f)$ is supported in $E_{1}=2 Q \backslash r_{0} Q$ and $\operatorname{dist}\left(E_{1}, \overline{\mathbf{S}_{\text {sing }}(w)}\right)>0$, hence

$$
\psi \operatorname{ext}_{Q}(f-\varphi f) \in B_{p_{1}, q_{1}}^{s_{1}}\left(E_{1}\right)=B_{p_{1}, q_{1}}^{s_{1}}\left(E_{1}, w\right)
$$

(in the sense of equivalent norms). We define

$$
\widetilde{\mathrm{T}} f=T\left(\psi\left(\varphi f+\operatorname{ext}_{Q}(f-\varphi f)\right)\right)
$$

where $T$ is the operator mentioned in Remark 3.2. It follows from Theorem 2.6, the extension property of Besov spaces defined on Lipschitz domains and (4.29) that $\widetilde{T}$ is a bounded linear operator from $B_{p_{1}, q_{1}}^{s_{1}}(Q, w)$ into $\widetilde{b}_{p_{1}, q_{1}}^{\sigma_{1}}(w)$ (with the constant $c$ depending on $Q$ and the size of supports of the wavelets.)

On the other hand, we put

$$
\widetilde{\mathrm{S}}(\lambda)=\operatorname{re}_{Q}(S(\lambda)),
$$

where $S$ is the operator from Remark 3.2 and $\mathrm{re}_{Q}: B_{p_{2}, q_{2}}^{s_{2}}\left(\mathbf{R}^{n}, w\right) \rightarrow B_{p_{2}, q_{2}}^{s_{2}}(Q, w)$ is a restriction operator. It should be clear by the construction that the diagram (4.27) 
is commutative. The operator $\widetilde{\mathrm{S}}$ is a bounded linear operator from $\widetilde{b}_{p_{2}, q_{2}}^{\sigma_{2}}(w)$ into $B_{p_{2}, q_{2}}^{s_{2}}(Q, w)$. Consequently,

$$
e_{k}\left(\mathrm{id}_{w w}^{Q}\right) \leq c e_{k}\left(\operatorname{Id}_{w w}^{Q}\right) \leq c e_{k}\left(\mathrm{id}: \widetilde{b}_{p_{1}, q_{1}}^{\sigma_{1}} \rightarrow \widetilde{b}_{p_{2}, q_{2}}^{\sigma_{2}}\right)
$$

and

$$
a_{k}\left(\operatorname{id}_{w w}^{Q}\right) \leq c a_{k}\left(\operatorname{Id}_{w w}^{Q}\right) \leq c a_{k}\left(\mathrm{id}: \widetilde{b}_{p_{1}, q_{1}}^{\sigma_{1}} \rightarrow \widetilde{b}_{p_{2}, q_{2}}^{\sigma_{2}}\right),
$$

cf. Remark 3.2. But

$$
e_{k}\left(\mathrm{id}: \widetilde{b}_{p_{1}, q_{1}}^{\sigma_{1}} \rightarrow \widetilde{b}_{p_{2}, q_{2}}^{\sigma_{2}}\right) \sim e_{k}\left(\mathrm{id}: \ell_{q_{1}}\left(2^{j \delta} \ell_{p_{1}}^{C 2^{n j}}\right) \rightarrow \ell_{q_{2}}\left(\ell_{p_{2}}^{C 2^{n j}}\right)\right) \leq c^{\prime} k^{-\frac{s_{1}-s_{2}}{n}},
$$

where

$$
\begin{aligned}
\ell_{q}\left(2^{j \delta} \ell_{p}^{C 2^{j n}}\right)= & \left\{\lambda=\left\{\lambda_{j, m}\right\}_{j, m}: \lambda_{j, m} \in \mathbf{C}, j, m \in \mathbf{N}_{0}, 0 \leq m \leq C 2^{j n}\right. \\
& \left.\left\|\lambda \mid \ell_{q}\left(2^{j \delta} \ell_{p}(w)\right)\right\|=\left(\sum_{j=0}^{\infty} 2^{j \delta q}\left(\sum_{m=0}^{C 2^{j n}}\left|\lambda_{j, m}\right|^{p}\right)^{\frac{q}{p}}\right)^{\frac{1}{q}}<\infty\right\}
\end{aligned}
$$

(appropriately modified if $q=\infty$ ). The final estimate in (4.34) is a consequence of the results in [22, Thm 4.14, Cor. 4.16] related to entropy numbers of the above sequence space embeddings. (Note that it is sufficient to take $\alpha>\delta$ in [22, Cor. 4.16] since $\ell_{q}\left(2^{j \delta} \ell_{p}^{C 2^{j n}}\right) \hookrightarrow \ell_{q}\left(2^{j \delta} \ell_{p}\left(w_{\alpha}\right)\right)$.) In a similar way we get

$$
a_{k}\left(\mathrm{id}: \widetilde{b}_{p_{1}, q_{1}}^{\sigma_{1}} \rightarrow \widetilde{b}_{p_{2}, q_{2}}^{\sigma_{2}}\right) \sim a_{k}\left(\mathrm{id}: \ell_{q_{1}}\left(2^{j \delta} \ell_{p_{1}}^{C 2^{n j}}\right) \rightarrow \ell_{q_{2}}\left(\ell_{p_{2}}^{C 2^{n j}}\right)\right) \leq c k^{-\varkappa}
$$

using results of [33].

Remark 4.13. It might be surprising at first glance that unlike in the one-sided weighted situation presented in Corollary 4.9(ii) we have in the double-sided weighted situation (4.23) the optimal outcome not only for entropy, but also for approximation numbers (4.25). The main trick, however, is the reduction to a corresponding unweighted setting (4.32), (4.33) which gives the correct upper estimates (the lower estimates (4.20) are clear in both situations). This recommends a refined study of embeddings of type

$$
\mathrm{id}: \widetilde{b}_{p_{1}, q_{1}}^{\sigma_{1}}(w) \hookrightarrow \widetilde{b}_{p_{2}, q_{2}}^{\sigma_{2}}
$$

and their corresponding approximation numbers. Our special example $w=w_{\alpha, \beta}$ given by (2.9) suggests that (4.20) indicates the asymptotic behaviour of the approximation numbers correctly, see also [17, Lemma 4.2].

\section{References}

[1] Bownik, M.: Atomic and molecular decompositions of anisotropic Besov spaces. - Math. Z. 250, 2005, 539-571.

[2] Bownik, M., and K.-P. Ho: Atomic and molecular decompositions of anisotropic TriebelLizorkin spaces. - Trans. Amer. Math. Soc. 358:4, 2006, 1469-1510.

[3] BuI, H.-Q.: Weighted Besov and Triebel spaces: Interpolation by the real method. - Hiroshima Math. J. 12:3, 1982, 581-605.

[4] Bui, H.-Q.: Characterizations of weighted Besov and Triebel-Lizorkin spaces via temperatures. - J. Funct. Anal. 55:1, 1984, 39-62. 
[5] Bui, H.-Q., M. Paluszyński, and M. H. Taibleson: A maximal function characterization of weighted Besov-Lipschitz and Triebel-Lizorkin spaces. - Studia Math. 119:3, 1996, 219-246.

[6] Bui, H.-Q., M. Paluszyński, and M. H. Taibleson: Characterization of the Besov-Lipschitz and Triebel-Lizorkin spaces. The case $q<1$. - J. Fourier Anal. Appl. 3, 1997, 837-846.

[7] Caetano, A.: About approximation numbers in function spaces. - J. Approx. Theory 94, 1998, 383-395.

[8] Carl, B., and I. Stephani: Entropy, compactness and the approximation of operators. Cambridge Univ. Press, Cambridge, 1990.

[9] Edmunds, D. E., and W. D. Evans: Spectral theory and differential operators. - Oxford Univ. Press, Oxford, 1987.

[10] Edmunds, D. E., and H. Triebel: Function spaces, entropy numbers, differential operators. - Cambridge Univ. Press, Cambridge, 1996.

[11] Falconer, K. J.: The geometry of fractal sets. - Cambridge Univ. Press, Cambridge, 1985.

[12] FARwig, R., and H. Sohr: Weighted $L^{q}$-theory for the Stokes resolvent in exterior domains. - J. Math. Soc. Japan 49:2, 1997, 251-288.

[13] Frazier, M., and S. Roudenko: Matrix-weighted Besov spaces and conditions of $\mathscr{A}_{p}$ type for $0<p \leq 1$. - Indiana Univ. Math. J. 53:5, 2004, 1225-1254.

[14] García-Cuerva, J., and J. L. Rubio de Francia. - Weighted norm inequalities and related topics. - North-Holland, Amsterdam, 1985.

[15] Haroske, D.: Approximation numbers in some weighted function spaces. - J. Approx. Theory 83:1, 1995, 104-136.

[16] Haroske, D. D., and I. Piotrowska: Atomic decompositions of function spaces with Muckenhoupt weights, and some relations to fractal analysis. - Math. Nachr. 281, 2008, 1476-1494.

[17] Haroske, D. D., and L. SkrzypczaK: Entropy and approximation numbers of embeddings of function spaces with Muckenhoupt weights, I. - Rev. Mat. Complut. 21, 2008, 135-177.

[18] Haroske, D. D., and L. Skrzypczak: Entropy numbers of embeddings of function spaces with Muckenhoupt weights, III. Some limiting cases. - J. Funct. Spaces Appl. (to appear).

[19] Haroske, D., and H. Triebel: Entropy numbers in weighted function spaces and eigenvalue distribution of some degenerate pseudodifferential operators I. - Math. Nachr. 167, 1994, 131156.

[20] Jonsson, A., and H. Wallin: Function spaces on subsets of $\mathbf{R}^{n}$. - Math. Rep. 2:1, 1984, $1-221$.

[21] KÜhn, Th., H.-G. Leopold, W. Sickel, and L. SkrzypcZaK: Entropy numbers of embeddings of weighted Besov spaces. - Constr. Approx. 23, 2006, 61-77.

[22] KÜhn, Th., H.-G. Leopold, W. Sickel, and L. Skrzypczak: Entropy numbers of embeddings of weighted Besov spaces II. - Proc. Edinb. Math. Soc. (2) 49, 2006, 331-359.

[23] KÜhn, Th., H.-G. Leopold, W. Sickel, and L. Skrzypczak: Entropy numbers of embeddings of weighted Besov spaces III. Weights of logarithmic type. - Math. Z. 255, 2007, $1-15$.

[24] Muckenhoupt, B.: Hardy's inequality with weights. - Studia Math. 44, 1972, 31-38.

[25] Muckenhoupt, B.: Weighted norm inequalities for the Hardy maximal function. - Trans. Amer. Math. Soc. 165, 1972, 207-226.

[26] Muckenhoupt, B.: The equivalence of two conditions for weight functions. - Studia Math. 49, 1972, 101-106.

[27] Mynbaev, K., and M. Otel'Baev: Weighting functional spaces and differential operators Spectrum. - Nauka, Moscow, 1988 (in Russian). 
[28] Pietsch, A.: Eigenvalues and s-numbers. - Akad. Verlagsges. Geest \& Portig, Leipzig, 1987.

[29] Roudenko, S.: Matrix-weighted Besov spaces. - Trans. Amer. Math. Soc. 355, 2002, 273-314.

[30] Roudenko, S.: Duality of matrix-weighted Besov spaces. - Studia Math. 160, 2004, 129-156.

[31] Rychkov, V.S.: Littlewood-Paley theory and function spaces with $A_{p}^{\ell o c}$ weights. - Math. Nachr. 224, 2001, 145-180.

[32] Sickel, W., and H. Triebel: Hölder inequalities and sharp embeddings in function spaces of $B_{p, q}^{s}$ and $F_{p, q}^{s}$ type. - Z. Anal. Anwendungen 14, 1995, 105-140.

[33] Skrzypczak, L.: On approximation numbers of Sobolev embeddings of weighted function spaces. - J. Approx. Theory 136, 2005, 91-107.

[34] Stein, E. M.: Harmonic analysis, real-variable methods, orthogonality, and oscillatory integrals. - Princeton Univ. Press, Princeton, 1993.

[35] Strömberg, J.-O., and A. Torchinsky: Weighted Hardy spaces. - Lecture Notes in Math. 1381, Springer, Berlin, 1989.

[36] Torchinsky, A.: Real-variable methods in harmonic analysis. - Academic Press, Orlando, FL, 1986.

[37] Triebel, H.: Theory of function spaces. - Birkhäuser, Basel, 1983.

[38] Triebel, H.: Theory of function spaces II. - Birkhäuser, Basel, 1992.

[39] Triebel, H.: Fractals and spectra. - Birkhäuser, Basel, 1997.

[40] Triebel, H.: Theory of function spaces III. - Birkhäuser, Basel, 2006.

Received 31 December 2009 